\title{
ENDOTRIVIAL MODULES FOR THE SPORADIC SIMPLE GROUPS AND THEIR COVERS
}

\author{
CAROLINE LASSUEUR AND NADIA MAZZA
}

\begin{abstract}
We investigate endotrivial modules for the sporadic simple groups and their covers, in a step towards the classification of endotrivial modules for quasi-simple groups. A main outcome of our study is the existence of torsion endotrivial modules with dimension greater than one for several sporadic groups with $p$-rank greater than one.
\end{abstract}

\section{INTRODUCTION}

Let $G$ be a finite group and $k$ a field of prime characteristic $p$ dividing the order of $G$. A $k G$-module $V$ is called endotrivial if $V \otimes V^{*} \cong k \oplus Q$, with $Q$ a projective $k G$-module. The tensor product over $k$ induces a group structure on the set of isomorphism classes of indecomposable endotrivial $k G$-modules, called the group of endotrivial modules and denoted $T(G)$. This group is finitely generated and it is of particular interest in modular representation theory as it forms an important part of the Picard group of self-equivalences of the stable category of finitely generated $k G$-modules. In particular the self-equivalences of Morita type are induced by tensoring with endotrivial modules.

Endotrivial modules have seen a considerable interest since defined by Dade in 1978 in [Dad78a, Dad78b] where he gives a classification for abelian $p$-groups, in which case $T(G)$ is cyclic generated by the module $\Omega(k)$. Since then a full classification has been obtained over $p$-groups (see [Car12] and the references therein). Contributions towards a general classification of endotrivial modules have already been obtained for several families of general finite groups ([CMN06, CMN09, CHM10, MT07, CMT11, CMT13, LMS13, NR12, Rob11]), however the problem of computing $T(G)$ for an arbitrary finite group $G$ remains open in general. In particular the problem of determining the structure of the torsion subgroup $T T(G)$ of $T(G)$ is the hardest part. In [Car12, Thm. A] J. Carlson states that in the known cases, but for few exceptions, $T T(G)=X(G)$, the group of one-dimensional $k G$-modules.

In this article, we study endotrivial modules for the sporadic simple groups and their covering groups, i.e. perfect groups $G$ such that $G / Z(G)$ is sporadic simple. This in all characteristics $p$ dividing the order of $G$. As a consequence we obtain a significant list of exceptions where $T T(G)$ is non-trivial, and a fortiori not isomorphic to $X(G)$.

Our first main result is the determination of the torsion-free rank of $T(G)$ for all covering groups $G$ of sporadic groups. Our second main result is the full structure of $T(G)$ for all covering groups of sporadic groups in characteristic 2. Our third main result concerns

Date: May 9, 2014.

1991 Mathematics Subject Classification. Primary 20C20; Secondary 20C34.

The first author gratefully acknowledges financial support by ERC Advanced Grant 291512 and SNF Fellowship for Prospective Researchers PBELP2_143516. 
proper covering groups, i.e. cases where $Z(G)$ is non-trivial: in any such case, we either prove that $T(G) \cong T(G / Z(G))$ via inflation, or compute $T(G)$ by explicit arguments. Our fourth main result is the full structure of the group $T(G)$ in all characteristics where the Sylow $p$-subgroups are cyclic, although in this case the main theoretical ingredients are provided by [MT07].

At this point we are left with the computation of the torsion subgroup $T T(G)$ in odd characteristic $p$ such that $p^{2}$ divides the order of $G$. In this paper we determine the full structure of $T T(G)$ in all characteristics for the following groups: $\mathrm{M}_{11}, \mathrm{M}_{12}, \mathrm{~J}_{1}, \mathrm{M}_{22}, \mathrm{~J}_{2}$, $\mathrm{M}_{23},{ }^{2} \mathrm{~F}_{4}(2)^{\prime}$, HS, $\mathrm{M}_{24}, \mathrm{McL}$, Suz, $\mathrm{Fi}_{22}, \mathrm{HN}, \mathrm{Ly}, \mathrm{J}_{4}$ and their covers. In the remaining cases, we do not obtain the full structure of $T T(G)$ for all $p|| G \mid$, but we give as much information as possible. Typically, in most missing cases we can give an upper bound in terms of abelian groups for $T T(G)$.

Our methods combine on the one hand traditional modular representation theory techniques such as the theory of vertices and sources (see [Car12]) and on the other hand character theory. More specifically we use the well-known fact that trivial source modules lift uniquely to characteristic zero and use the newly obtained characterisation of the torsion subgroup $T T(G)$ via ordinary characters of the group $G$ (see Theorem 2.11). In particular this approach allows us to compute Green correspondence at the level of ordinary characters, rather than at the level of modules, allowing us to make computer calculations via the GAP Character Table Librairies even for large sporadic groups. Whenever possible we will provide the characters afforded by trivial source endotrivial module, so that using the known decomposition matrices, one can recover the composition factors of these modules. In addition, we rely on a recently developed approach by Balmer ([Bal13]) which uses weak $H$-homomorphisms. Leaving aside the details, Balmer's method is useful in helping to find the trivial source endotrivial modules, though it does not provide a complete answer in general.

Our paper is organised as follows. In Sections 2 and 3, we sum up useful results on endotrivial modules and the sporadic groups. In section 4 , we determine the structure of $T(G)$ in characteristic 2. For odd characteristics, in Section 5 we determine the torsionfree rank of $T(G)$, and in Section 6 the torsion subgroup $T T(G)$ when we have obtained its full structure. We collect the results in Section 7 in Table 3 if $p^{2}|| G \mid$ and in Table 5 if the Sylow $p$-subgroups are cyclic.

Acknowledgments. The first author would like to thank Prof. Dr. Gunter Malle and the FB Mathematik of the TU Kaiserslautern for hosting her as an SNF Fellow during the academic year 2013-2014, as well as the department of Mathematics of the University of Lancaster for supporting her visit in October 2013. The second author is thankful to the FB Mathematik of the TU Kaiserslautern for hosting her for a short visit in Spring 2013, during which this project started. Both authors are sincerely indebted to Jon Carlson for his help with computer calculations.

\section{Endotrivial MOdUles: BACKGROUND RESUlts}

Throughout, unless otherwise stated, we let $G$ be a finite group, $P$ a Sylow $p$-subgroup of $G$, for some prime $p$ dividing the order of $G$, and $k$ an algebraically closed field of characteristic $p$. We write $N_{G}(P)$ for the normaliser of $P$ in $G$ and we adopt the same notation and conventions as in [Thé95]. In particular, all $k G$-modules are left modules 
and finitely generated. In addition, for a $k G$-module $M$, we put $M^{*}=\operatorname{Hom}_{k}(M, k)$ for the $k$-dual of $M$ and $\otimes=\otimes_{k}$. Also, $X(G)$ denotes the group of isomorphism classes of 1-dimensional $k G$-modules.

\subsection{Definitions and elementary properties.}

Definition 2.1. A $k G$-module $M$ is endotrivial if $\operatorname{End}_{k}(M) \cong k \oplus$ (proj) as $k G$-modules, where (proj) denotes some projective $k G$-module.

Here are the main basic properties of endotrivial modules.

Lemma 2.2. Let $M$ be a $k G$-module.

(1) $M$ is endotrivial if and only if $M$ splits as the direct sum $M_{0} \oplus$ (proj) for an indecomposable endotrivial $k G$-module $M_{0}$, unique up to isomorphism.

(2) The relation

$$
M \sim N \Longleftrightarrow M_{0} \cong N_{0}
$$

on the class of endotrivial $k G$-modules is an equivalence relation and every equivalence class contains a unique indecomposable module up to isomorphism.

(3) If $M$ is endotrivial, then $M \downarrow_{H}^{G}$ is endotrivial for any subgroup $H$ of $G$. Moreover if $H \geq P \in \operatorname{Syl}_{p}(G)$, then $M$ is endotrivial if and only if $M \downarrow_{H}^{G}$ is.

(4) $M$ is endotrivial if and only if $M^{*}$ is endotrivial.

(5) Given two endotrivial $k G$-modules $M, N$, then $M \otimes N$ is endotrivial.

(6) Let $0 \longrightarrow M \longrightarrow Q \longrightarrow N \longrightarrow 0$ be a short exact sequence of $k G$-modules with $Q$ projective. Then $M$ is endotrivial if and only if $N$ is endotrivial.

(7) If $M$ is endotrivial, then

$$
\operatorname{dim}_{k}(M) \equiv\left\{\begin{array}{lll} 
\pm 1 & \left(\bmod |G|_{p}\right) & \text { if } p \text { is odd } \\
\pm 1 & \left(\bmod \frac{1}{2}|G|_{2}\right) & \text { if } p=2
\end{array}\right.
$$

where $|G|_{p}$ is the order of a Sylow p-subgroup of $G$. Also, if $M$ is moreover a trivial source module, then $\operatorname{dim}_{k}(M) \equiv 1\left(\bmod |G|_{p}\right)$.

Part (2) is equivalent to saying that two endotrivial modules $M, N$ are equivalent if and only is they are isomorphic in the stable module category of $k G$. This leads us to the following definition:

Definition 2.3. The set $T(G)$ of equivalence classes of endotrivial $k G$-modules resulting from the relation $\sim$ of Lemma 2.2(2) forms an abelian group for the composition law

$$
[M]+[N]:=[M \otimes N]
$$

The zero element of $T(G)$ is the class $[k]$ of the trivial module, and the additive inverse of $[M]$ is $-[M]=\left[M^{*}\right]$. The group $T(G)$ is called the group of endotrivial $k G$-modules.

If $P_{*} \rightarrow M$ is a minimal projective resolution of a $k G$-module $M$, we denote by $\Omega^{n}(M)$ the $(n-1)$-st kernel of the differentials, and similarly $\Omega^{-n}(M)$ is the $(n-1)$-st cokernel in an injective resolution $M \hookrightarrow I_{*}$ of $M$. By convention, $\Omega^{0}(M)$ is the projective-free part of $M$ and $\Omega(M)=\Omega^{1}(M)$. Thus part (6) in Lemma 2.2 provides us with an important family of endotrivial modules, namely the modules $\Omega^{n}(k)$ for $n \in \mathbb{Z}$. Henceforth we write $\Omega$ for the equivalence class of $\Omega(k)$ in $T(G)$. For $m, n \in \mathbb{Z}, \Omega^{n}(k) \otimes \Omega^{m}(k) \cong \Omega^{m+n}(k) \oplus$ (proj), hence $\langle\Omega\rangle \leq T(G)$ is a cyclic subgroup, and, unless $G$ has $p$-rank one, $\langle\Omega\rangle \cong \mathbb{Z}$. 
2.2. Previously known results. The abelian group $T(G)$ is known to be finitely generated and so we can write

$$
T(G)=T T(G) \oplus T F(G)
$$

where $T T(G)$ is the torsion subgroup of $T(G)$ and $T F(G)$ is a torsion-free direct sum complement of $T T(G)$ in $T(G)$. In particular $T T(G)$ is finite. The rank of $T F(G)$ is the torsion-free rank of $T(G)$ and it is written $n_{G}$. It is determined in [CMN06].

Lemma 2.4 ([CMN06]). The torsion-free rank of $T(G)$ is equal to the number of conjugacy classes of maximal elementary abelian p-subgroups of rank 2 if $G$ has p-rank 2 , or that number plus one if $G$ has p-rank greater than 2.

The next three results characterising the torsion-free rank of $T(G)$ are at the crossroad of local group structure and representation theory.

Theorem 2.5 ([GM10, Mac70]). Let $G$ be a finite p-group. Suppose that $G$ has a maximal elementary abelian $p$-subgroup of order $p^{2}$. Then $G$ has $p$-rank at most $p$ if $p$ is odd, or at most 4 if $p=2$.

For completeness, the case $p=2$ is due to A. MacWilliams (often coined the fourgenerator theorem), and for odd primes it is work of G. Glauberman and the second author.

Corollary 2.6. Assume the p-rank of the group $G$ is greater $p$ if $p$ is odd, or greater than 4 if $p=2$. Then $T(G)$ has torsion-free rank one.

Theorem 2.7 ([Car07, Maz08]). Let $G$ be a finite p-group. Then $n_{G} \leq p+1$ if $p$ is odd, or at most $n_{G} \leq 5$ if $p=2$. Moreover, both upper bounds are optimal.

Optimality of the bound $n_{G}$ for $p$ odd is for instance obtained with $G$ an extraspecial $p$-group of order $p^{3}$ and exponent $p$ if $p$ is odd, whereas for $p=2$, we can take for $G$ an extraspecial 2-group of order 32 of the form $Q_{8} * D_{8}$.

We now present a collection of useful results, which have been proven in previous articles on endotrivial modules, see [Car12] and the references therein. Recall that a subgroup $H$ of $G$ is strongly $p$-embedded if $p$ divides the order of $H$ but $p$ does not divide the order of $H \cap{ }^{g} H$ for each $g \in G-H$. In particular, $N_{G}(P)$ is strongly $p$-embedded in $G$ if and only if the Sylow $p$-subgroup $P$ is a trivial intersection (T.I. hereafter) subset of $G$. Also, if $H$ is strongly $p$-embedded in $G$, then $H \geq N_{G}(P)$.

Useful information on $T(G)$ can sometimes be gathered by comparison with known endotrivial modules for subgroups of $G$. For instance, if $H$ is a subgroup of $G$, the restriction along the inclusion induces a group homomorphism $\operatorname{Res}_{H}^{G}: T(G) \rightarrow T(H)$. In contrast, the induction $\operatorname{Ind}_{H}^{G}(M)$ of an endotrivial $k H$-module $M$ needs not be endotrivial for $G$.

Lemma 2.8 (Omnibus Lemma). Let $H$ be a subgroup of $G$ containing the normaliser $N_{G}(P)$ of a Sylow p-subgroup $P$ of $G$.

(1) If $G$ has p-rank at least 2 , then $\Omega$ generates an infinite cyclic direct summand of $T F(G)$ and can be chosen as part of a set of generators for $T F(G)$. In particular, if $T(G)$ has torsion-free rank 1 , then $T F(G)=\langle\Omega\rangle$. 
(2) The restriction map $\operatorname{Res}_{H}^{G}: T(G) \longrightarrow T(H)$ is injective. More precisely, if $M$ is an indecomposable endotrivial $k G$-module and if $M \downarrow_{H}^{G}=L \oplus$ (proj) where $L$ is an indecomposable $k H$-module, then $M$ is the $k G$-Green correspondent of $L$ and $L$ is endotrivial.

(3) Assume that for all $x \in G$ the subgroup ${ }^{x} P \cap P$ is non trivial. Then, the kernel of the restriction map $\operatorname{Res}_{P}^{G}: T(G) \rightarrow T(P)$ is generated by the isomorphism classes of 1-dimensional $k G$-modules. In particular, this holds whenever $G$ has a non-trivial normal p-subgroup.

(4) If $H$ is strongly $p$-embedded in $G$, then $\operatorname{Res}_{H}^{G}: T(G) \longrightarrow T(H)$ is an isomorphism, the inverse map being induced by the induction $\operatorname{Ind}_{H}^{G}$.

(5) If $P=N_{G}(P)$ is neither cyclic, nor semi-dihedral, nor generalised quaternion, then $T T(G)=\{[k]\}$.

Lemma 2.8 shows that a large part of $T T(G)$ is generated by the (stable) isomorphism classes of trivial source endotrivial modules. Indeed, unless a Sylow $p$-subgroup $P$ of $G$ has normal $p$-rank one, then $T(P)$ is torsion-free and so $T T(G)$ is detected by restriction to $P$ in the sense of Lemma $2.8(2)$.

Many of the Sylow $p$-subgroups of sporadic groups are cyclic for $p>2$. In fact if $G$ is quasi-simple with $G / Z(G)$ sporadic simple and $P \in \operatorname{Syl}_{p}(G)$ is cyclic, then $P \cong C_{p}$ and $p \neq 2$. So [MT07, Theorem 3.2] describes the structure of $T(G)$ in this case.

Theorem 2.9. [MT07] Let $G$ be a finite group with a non-trivial cyclic Sylow p-subgroup $P$ with $|P|>2$. Let $Z$ be the unique subgroup of $P$ of order $p$ and let $H=N_{G}(Z)$. Then:

(1) $T(G)=\left\{\left[\operatorname{Ind}_{H}^{G}(M)\right] \mid[M] \in T(H)\right\} \cong T(H)$.

(2) There is an exact sequence

$$
0 \longrightarrow X(H) \longrightarrow T(H) \stackrel{\operatorname{Res}_{P}^{H}}{\longrightarrow} T(P) \longrightarrow 0
$$

where $T(P) \cong \mathbb{Z} / 2$. This sequence splits if and only if $\left[\Omega_{H}^{2}(k)\right]$ is a square in $X(H)$. In particular, this sequence splits if the index $e:=\left|N_{G}(Z): C_{G}(Z)\right|$ is odd.

2.3. Character theory for endotrivial modules. Finally we recall known results needed for our investigations which stem from ordinary character theory.

Theorem 2.10. [LMS13, Theorem 1.3 and Corollary 2.3] Let $(K, \mathcal{O}, k)$ be a splitting p-modular system. Let $V$ be an endotrivial $k G$-module. Then

(1) $V$ is liftable to an endotrivial $\mathcal{O} G$-lattice.

(2) Moreover, if $V$ is liftable to a $\mathbb{C} G$-module affording the character $\chi$, then $|\chi(g)|=1$ for every p-singular element $g \in G$.

Further, if a $k G$-module $M$ is the $k G$-Green correspondent of a 1-dimensional $k N_{G}(P)$ module, then $M$ is a trivial source module and it lifts uniquely to a trivial source $\mathcal{O} G$ lattice $\hat{M}$. Denote by $\chi_{\hat{M}}$ the ordinary character afforded by $\hat{M}$. Then endotriviality for $M$ can be read from the character table of $G$ as follows:

Theorem 2.11. [LM14, Theorem 2.2] Let $P \in S l_{p}(G)$ and let $M$ be the $k G$-Green correspondent of a 1-dimensional $k N_{G}(P)$-module. Then $M$ is endotrivial if and only if $\chi_{\hat{M}}(x)=1$ for all p-elements $x \in G \backslash\{1\}$. 
The latter Proposition relies on the following result by Green, Landrock and Scott on character values of trivial source modules, which we will also implicitly extensively use in computations in Sections 4 and 6.

Lemma 2.12. [Lan83, Part II, Lemma 12.6] Let $M$ be an indecomposable trivial source $k G$-module and $x \in G$ a p-element. Then,

(1) $\chi_{\hat{M}}(x) \geq 0$ is an integer (corresponding to the multiplicity of the trivial $k\langle x\rangle$ module as a direct summand of $\left.M \downarrow_{\langle x\rangle}^{G}\right)$;

(2) $\chi_{\hat{M}}(x) \neq 0$ if and only if $x$ belongs to a vertex of $M$.

\section{SPORADIC GROUPS AND THEIR COVERS}

3.1. Notation and terminology. We call sporadic simple any of the 26 standard sporadic simple groups together with the Tits simple group ${ }^{2} \mathrm{~F}_{4}(2)^{\prime}$. We use the Atlas [CCN $\left.{ }^{+} 85\right]$ notation:

$A \times B$ denotes a direct product of $A$ and $B$;

$A$. $B$ or $A B$ denotes a group having a normal subgroup isomorphic to $A$ with corresponding quotient isomorphic to $B$;

$A: B$ denotes a split extension of $A$ by $B$;

$A * B$ denotes a central product of $A$ by $B$;

$m$ denotes a cyclic group of order $m$;

$p^{n}$ denotes an elementary abelian $p$-group of order $p^{n}$;

$p_{+}^{1+2 n}$ where $p$ is an odd prime, denotes an extraspecial group of order $p^{1+2 n}$ and exponent $p$;

$D_{2^{n}}$ denotes a dihedral 2-group of order $2^{n}$;

$S D_{2^{n}}$ denotes a semi-dihedral 2-group of order $2^{n}$;

$Q_{8}$ denotes a quaternion 2-group of order 8;

$\mathfrak{S}_{n}$ denotes the symmetric group on $n$ letters;

$\mathfrak{A}_{n}$ denotes the alternating group on $n$ letters.

If more than one symbol is used, we read them left to right, that is we write $A . B: C$ for $(A . B): C$. In addition $Z(G)$ denotes the centre of $G$ and $G^{\prime}=[G, G]$ the commutator subgroup of $G$. Also, a $p^{\prime}$-group for a prime $p$ is a group of order not divisible by $p$.

3.2. Covering groups and inflation. A finite group $G$ is called quasi-simple if $G$ is a perfect central extension of a simple group. If $G$ is quasi-simple, then $H:=G / Z(G)$ is simple and $G$ is a perfect central extension of $H$. We then call $G$ a covering group of $H$. According to Notation $3.1, m . H$ denotes a covering group of a group $H$, which is a central extension of $H$ by a cyclic group of order $m$. The integer $m$ is called the degree of the central extension. If $\ell$ is a prime dividing $m$, then there exists a covering group $K$ of $H$ such that $m . H$ is a central extension of $K$ of degree $\ell$.

Lemma 3.1. Let $1 \longrightarrow A \longrightarrow G \stackrel{\pi}{\longrightarrow} H \longrightarrow 1$ be a central extension of $H$ by $A$ and $P, Q$ Sylow p-subgroups of $G$ and $H$ respectively. The following hold.

(1) $X\left(N_{H}(Q)\right)$ is isomorphic to a quotient group of $X\left(N_{G}(P)\right)$.

(2) If $p$ divides $|A|$ and $T T(P)=\{[k]\}$, then $T T(G)=\{[k]\}$. 
(3) If $p$ does not divide $|A|$, then the torsion-free rank of $T(H)$ equals the torsion-free rank of $T(G)$.

Recall that $T(P)$ is torsion-free if and only if $P$ is neither cyclic, generalised quaternion nor semi-dihedral.

Proof.

(1) By assumption, $\pi$ induces surjective homomorphisms $N_{G}(P) \stackrel{\pi}{\longrightarrow} N_{H}(Q)$ with kernel $A$, and $P N_{G}(P)^{\prime} \stackrel{\pi}{\longrightarrow} Q N_{H}(Q)^{\prime}$ with kernel $O_{p}(A)\left(A \cap N_{G}(P)^{\prime}\right)$. So $\pi$ induces a surjective homomorphism $N_{G}(P) / P N_{G}(P)^{\prime} \rightarrow N_{H}(Q) / Q N_{H}(Q)^{\prime}$ with kernel isomorphic to $A / O_{p}(A)\left(A \cap N_{G}(P)^{\prime}\right)$. Therefore $X\left(N_{H}(Q)\right)$ is isomorphic to a quotient of $X\left(N_{G}(P)\right)$.

(2) Since $T(P)$ is torsion-free, then $T T(G)$ is generated by the classes of the trivial source endotrivial modules. Now $O_{p}(G)=O_{p}(A)>1$ implies that such module must have dimension 1 , and because $G$ is perfect, only the trivial module has dimension 1.

(3) The torsion-free ranks of $T(G)$ and $T(H)$ are determined by the number of conjugacy classes of maximal elementary abelian subgroups of rank 2 . The claim follows from the fact $A$ is central in $G=A . H$.

When investigating endotrivial modules we often rely on an operation called inflation. Namely, if $N$ is a normal subgroup of the group $G$, the restriction along the natural projection map $G \rightarrow G / N$ makes a $k[G / N]$-module $V$ into a $k G$-module $\operatorname{Inf}_{G / N}^{G}(V)$ on which $N$ acts trivially. This operation is the inflation from $G / N$ to $G$. If $p \nmid|N|$, then inflation of an endotrivial $k[G / N]$-module is an endotrivial $k G$-module. Moreover, if $N$ is central, independently from the order of $N$, inflation commutes with Green correspondence in the following sense.

Lemma 3.2. Let $G$ be a finite group and let $N$ be a normal subgroup of $G$.

(1) Let $M$ be an endotrivial $k[G / N]$-module with $\operatorname{dim}_{k}(M)>1$. Then $\operatorname{Inf}_{G / N}^{G}(M)$ is an endotrivial $k G$-module if and only if $p$ does not divide $|N|$. In particular, if $p$ does not divide $|N|$, then inflation induces a well-defined group homomorphism

$$
\operatorname{Inf}_{G / N}^{G}: T(G / N) \rightarrow T(G):[M] \mapsto\left[\operatorname{Inf}_{G / N}^{G}(M)\right] .
$$

(2) Assume that $N \leq Z(G)$ and let $P \in \operatorname{Syl}_{p}(G)$. Let $V$ be a $k G$-module with vertex $P$ such that $V=\operatorname{Inf}_{G / N}^{G}(\bar{V})$ for some $k[G / N]$-module $\bar{V}$. Let $\Gamma(-)$ and $\bar{\Gamma}(-)$ denote the Green correspondence from $G$ to $N_{G}(P)$ and from $G / N$ to $N_{G}(P) / N$ respectively. Then $\operatorname{Inf}_{N_{G}(P) / N}^{N_{G}(P)}(\bar{\Gamma}(\bar{V})) \cong \Gamma\left(\operatorname{Inf}_{G / N}^{G}(\bar{V})\right)$.

Proof.

(1) Set $H:=G / N$ and let $P, Q$ be Sylow $p$-subgroups of $G$ and $H$ respectively. If $p$ divides $|N|$, then $1<O_{p}(N) \leq P$ acts trivially on $\operatorname{Inf}_{H}^{G}(L)$ for every projective $k H$-module $L$. Therefore it follows by definition that the inflation $\operatorname{Inf}_{H}^{G}(V)$ of an endotrivial $k H$-module $V$ is not an endotrivial $k G$-module. Now suppose that $p$ 
does not divide $|N|$, choose an isomorphism $\phi: P \rightarrow Q$ and denote by $\operatorname{Res}_{\phi}$ the restriction along $\phi$ of a $k Q$-module. Then, because $\operatorname{Res}_{P}^{G} \operatorname{Inf}_{H}^{G}(M) \cong \operatorname{Res}_{\phi} \operatorname{Res}_{Q}^{H}(M)$ for any $k H$-module $M$, by Lemma 2.2(3) inflation preserves endotrivial modules. The claim follows.

(2) See [DK09, Proposition 2.9].

3.3. T.I. Sylow $p$-subgroups. We end this section with the list of the finite simple groups which have noncyclic trivial intersection (T.I.) Sylow $p$-subgroups. Recall that $P \subset G$ is T.I. if $P \cap{ }^{g} P=\{1\}$ for any $g \in G \backslash N_{G}(P)$. In particular, if $|P|$ is prime, then $P$ is T.I..

Theorem 3.3. [Mic91, Proposition 4.6] Let $G$ be a nonabelian simple group with a noncyclic T.I. Sylow p-subgroup P. Then $G$ is isomorphic to one of the following groups.

(1) $\mathrm{L}_{2}(q)$ where $q=p^{n}$ with $n \geq 2$.

(2) $\mathrm{U}_{3}\left(q^{2}\right)$ where $q$ is a power of $p$.

(3) ${ }^{2} \mathrm{~B}_{2}\left(2^{2 m+1}\right)$ and $p=2$.

(4) ${ }^{2} \mathrm{G}_{2}\left(3^{2 m+1}\right)$ with $m \geq 1$ and $p=3$.

(5) $\mathrm{L}_{3}(4)$ or $\mathrm{M}_{11}$ and $p=3$.

(6) ${ }^{2} \mathrm{~F}_{4}(2)^{\prime}$ or $\mathrm{McL}$ and $p=5$.

(7) $\mathrm{J}_{4}$ and $p=11$.

We are now ready to combine both Sections 2 and 3 in order to investigate endotrivial modules for the sporadic groups and their covers. We start by looking at the case $p=2$ and whenever we think it more appropriate, we use the algebra softwares MAGMA [BCP97] or GAP [GAP13, Bre12]. For the clarity of exposure, we summarise the results in the Tables 3 and 5 in Section 7.

\section{ENDotrivial MOdUles FOR SPORADIC GROUPS IN CHARACTERISTIC 2}

In this section, we prove the results stated in Section 7 for a field $k$ of characteristic 2 . The 2-local structure of simple groups has thoroughly been analysed and important results shown. In particular, by a celebrated result due to Brauer and Suzuki it is well-known that a Sylow 2-subgroup of a simple group cannot be generalised quaternion ([BS59]), while [Suz86, Corollary 2 p. 144] asserts that a Sylow 2-subgroup of a nonabelian simple group cannot be cyclic. In view of these results it seems reasonable to believe that if $G$ is a nonabelian simple group then $T(G)$ has torsion-free rank one in characteristic 2 . We can prove slightly better.

Proposition 4.1. Let $G$ be a finite quasi-simple group with $G / Z(G)$ sporadic simple, then the torsion-free rank of $T(G)$ is one, that is, $T F(G)=\langle\Omega\rangle \cong \mathbb{Z}$.

Proof. Using Theorems 2.5 and 2.7, we need to show that if $G$ has a nonabelian Sylow 2-subgroup $P$ of rank 2, 3 or 4, then $P$ is either semi-dihedral, or $P$ has rank greater than 2 and no maximal elementary abelian subgroup of order 4. By inspection of [GLS98, Table 5.6.1], we are left with the following groups. If the 2-rank is 2 , then $G=\mathrm{M}_{11}$ and has a semi-dihedral Sylow 2-subgroup of order 16. It follows from [CMT13, Thm. 6.5] that $n_{G}=1$. Otherwise $G$ is one of $\mathrm{M}_{12}, 2 . \mathrm{M}_{12}, \mathrm{M}_{22}, 4 . \mathrm{M}_{22}, 3 . \mathrm{M}_{22}, 12 . \mathrm{M}_{22}, \mathrm{M}_{23}, \mathrm{~J}_{1}, \mathrm{~J}_{2}, 2 . \mathrm{J}_{2}$, 
$J_{3}, 3 . \mathrm{J}_{3}, \mathrm{Co}_{3}, \mathrm{HS}, \mathrm{McL}, 3 . \mathrm{McL}$, Ly, O'N, 3.O'N and its 2-rank is 3 or 4 . A routine verification of each of these cases shows that a Sylow 2-subgroup of $G$ has no maximal elementary abelian subgroup of order 4 .

By Lemma 2.8 and the specific 2-local structure of sporadic simple groups and their covers, the torsion subgroup of $T(P)$ is often trivial, and the Sylow 2-subgroups selfnormalising. This leads us to our next result.

Lemma 4.2. If $G$ is one of the groups $\mathrm{M}_{12}, 2 . \mathrm{M}_{12}, \mathrm{M}_{22}, 2 . \mathrm{M}_{22}, 4 . \mathrm{M}_{22}, 6 . \mathrm{M}_{22}, 12 . \mathrm{M}_{22}$, 2. $\mathrm{J}_{2}, \mathrm{M}_{23}, \mathrm{M}_{24}, \mathrm{HS}, 2 . \mathrm{HS}, \mathrm{McL}, \mathrm{He}, \mathrm{Ru}, 2 . \mathrm{Ru}, \mathrm{O}^{\prime} \mathrm{N}, \mathrm{Co}_{3}, \mathrm{Co}_{2}, \mathrm{Fi}_{22}, 2 . \mathrm{Fi}_{22}, 6 . \mathrm{Fi}_{22}, \mathrm{Ly}$, Th, $\mathrm{Fi}_{23}, \mathrm{Co}_{1}, 2 . \mathrm{Co}_{1}, \mathrm{~J}_{4}, \mathrm{Fi}_{24}^{\prime}, \mathrm{B}, 2 . \mathrm{B}, \mathrm{M}$, or ${ }^{2} \mathrm{~F}_{4}(2)^{\prime}$, then $T T(G)=\{[k]\}$.

Proof. Let $P \in \operatorname{Syl}_{p}(G)$. If $G$ is one of $\mathrm{M}_{12}, 2 . \mathrm{M}_{12}, \mathrm{M}_{22}, 2 . \mathrm{M}_{22}, 4 . \mathrm{M}_{22}, \mathrm{M}_{23}, \mathrm{M}_{24}, \mathrm{HS}$, 2.HS, McL, He, Ru, 2.Ru, 2.Suz, 6.Suz, O’N, $\mathrm{Co}_{3}, \mathrm{Co}_{2}, \mathrm{Fi}_{22}, 2 . \mathrm{Fi}_{22}, 6 . \mathrm{Fi}_{22}$, Ly, Th, $\mathrm{Fi}_{23}$, $\mathrm{Co}_{1}, 2 . \mathrm{Co}_{1}, \mathrm{~J}_{4}, \mathrm{Fi}_{24}^{\prime}, \mathrm{B}, 2 . \mathrm{B}, \mathrm{M}$, or ${ }^{2} \mathrm{~F}_{4}(2)^{\prime}$, then one can read from [Wil98, Table 1] that $P$ is self-normalising. Therefore, $T T(G)=\{[k]\}$ by Lemma 2.8. If $G$ is one of $6 \cdot \mathrm{M}_{22}$, $12 . \mathrm{M}_{22}, 2 . \mathrm{J}_{2}, 2 . \mathrm{Suz}, 6 . \mathrm{Suz}$, or $6 . \mathrm{Fi}_{22}$ then $G$ has a non-trivial normal 2-subgroup and so $T T(G)=\{[k]\}$ by Lemma 3.1.

Lemma 4.3. If $G$ is one of the 3 -fold covers $3 . \mathrm{M}_{22}$, 3.Suz, 3.O'N, 3. $\mathrm{Fi}_{22}$, or $3 . \mathrm{Fi}_{24}^{\prime}$, then $G$ has no faithful endotrivial module. Therefore $T(G) \cong T(G / Z(G)) \cong \mathbb{Z}$ via inflation.

Proof. Assume $M$ is a faithful endotrivial $k G$-module and let $\chi$ be an ordinary character of $G$ belonging to the lift of $M$. Then all irreducible constituents of $\chi$ lie in a common faithful block of $k G$ and by Theorem 2.10 the values of $\chi$ on 2-singular conjugacy classes are roots of unity. However, it can be read from the GAP character table libraries [Bre12] (or the Atlas $\left[\mathrm{CCN}^{+} 85\right]$ ) that $G$ has a 2 -singular class $C$ such that for all faithful characters $\psi \in \operatorname{Irr}(G)$ we have $\psi(c) \in m \mathbb{Z}$ with $m \in \mathbb{Z} \backslash\{ \pm 1\}, c \in C$. Hence a contradiction. More precisely for $G=3 . \mathrm{M}_{22}$, take $(m, C)=(2,6 c)$, for $G=3$. Suz take $(m, C)=(0,6 q)$, for $G=3 . \mathrm{O}^{\prime} \mathrm{N}$ take $(m, C)=(0,6 c)$, for $G=3$. Fi $\mathrm{i}_{22}$ take $(m, C)=(0,6 w)$, for $G=3$. $\mathrm{Fi}_{24}^{\prime}$ take $(m, C)=(0,6 a d)$. (Notation for conjugacy classes is that of GAP [Bre12].)

We are left with the computation of the torsion subgroup $T T(G)$ in characteristic 2 for the following groups: $\mathrm{M}_{11}, \mathrm{~J}_{1}, \mathrm{~J}_{2}, \mathrm{~J}_{3}, 3 . \mathrm{J}_{3}, 3 . \mathrm{McL}$, Suz, HN. We proceed case by case.

\subsection{The Mathieu group $\mathrm{M}_{11}$.}

The group $G=\mathrm{M}_{11}$ has a semi-dihedral Sylow 2-subgroup $P$ of order 16 and $P=$ $N_{G}(P)$. From [CMT13, Theorems 6.4 and 6.5], we know that $T\left(\mathrm{M}_{11}\right)=\langle\Omega,[M]\rangle \cong \mathbb{Z} \oplus$ $\mathbb{Z} / 2$ where $M$ is the $k G$-Green correspondent of the translated relative syzygy $\Omega\left(\Omega_{P / C}(k)\right)$ for a noncentral subgroup $C$ of $P$ of order 2. Moreover $M$ is self-dual. This module was explicitly described by Kawata and Okuyama [Kaw93, Thm. 5.1] as the relative syzygy $M \cong \Omega\left(\Omega_{G / C}(k)\right)$. Computations with MAGMA show that $\operatorname{dim}_{k}(M)=41$.

\subsection{The Janko groups: $\mathrm{J}_{1}, \mathrm{~J}_{2}, \mathrm{~J}_{3}$ and $3 . \mathrm{J}_{3}$.}

Let $G=\mathrm{J}_{1}$ and $P$ a Sylow 2 -subgroup of $G$. Then $N_{G}(P) \cong P: 7: 3\left(\operatorname{cf}\left[\mathrm{CCN}^{+} 85\right]\right)$ and $X\left(N_{G}(P)\right) \cong \mathbb{Z} / 3$ is generated by a 1-dimensional $k N$-module $\lambda$ with $\lambda^{\otimes 3}=k$. The socle and Loewy series of the $k G$-Green correspondent $\Gamma(\lambda)$ of $\lambda$ have been computed in [LM78, Cor 5.8], from which we gather that $\Gamma(\lambda)$ has dimension 133 and affords the ordinary 
character $\chi_{12}$. By Lemma 2.2(7), $\Gamma(\lambda)$ cannot be endotrivial as $133 \not \equiv 1(\bmod |P|)$. Therefore $T T\left(\mathrm{~J}_{1}\right)=\{[k]\}$.

The groups $\mathrm{J}_{2}$ and $\mathrm{J}_{3}$ have very similar 2-local structure. Put $G$ for any of these Janko groups. The group $G$ has a Sylow 2-subgroup $P=\left(Q_{8} * D_{8}\right): 2^{2}$ of order $2^{7}$ and 2 rank 4. The index of $P$ in its normaliser $N$ is 3 , so that $X(N) \cong \mathbb{Z} / 3$. Moreover $G$ has a maximal subgroup $H$ containing $N$ and $H$ is isomorphic to $\left(Q_{8} * D_{8}\right): \mathfrak{A}_{5}$. The permutation module $k[H / P]$ gives all the indecomposable trivial source $k H$-modules up to isomorphism and MAGMA computations give that $k[H / P]$ splits into a direct sum of modules with dimensions $1,4,5,5$. Therefore $k$ is the only torsion endotrivial $k H$-module and a fortiori $T T(G)=\{[k]\}$ for $G=\mathrm{J}_{2}$ and $G=\mathrm{J}_{3}$. For $G=3 . \mathrm{J}_{3}$ we read from $\left[\mathrm{CCN}^{+} 85\right]$ that $G$ has a maximal subgroup $C(2 A)$ which is the centraliser of the central involution of $P$. Using MAGMA, we obtain that $C(2 A) \cong 3 \times\left(Q_{8} * D_{8}\right) \cdot \mathfrak{A}_{5}$ and its derived subgroup is $\left(Q_{8} * D_{8}\right) \cdot \mathfrak{A}_{5}$. Therefore $X(C(2 A)) \cong \mathbb{Z} / 3$ and $T T(C(2 A)) \cong \mathbb{Z} / 3$. Referring the reader to the method developed in [Bal13], as explained in Section 6, a further MAGMA computation shows that $T T\left(3 . \mathrm{J}_{3}\right) \cong \mathbb{Z} / 3$. In other words the Green correspondents of the two non-trivial 1-dimensional $k[C(2 A)]$-modules are endotrivial.

4.3. The group 3.McL. Let $G=3$.McL and $P$ a Sylow 2 -subgroup of $G$. Then $|P|=2^{7}$ and we have $X\left(N_{G}(P)\right) \cong \mathbb{Z} / 3$ since a Sylow 2-subgroup of McL is self-normalising. Because $T T(\mathrm{McL})=\{[k]\}$ by Lemma 4.2, a non-trivial trivial source endotrivial $k G$ module must be faithful. We see from the GAP character table librairies [Bre12] that $G$ has three 2-blocks of full defect: the principal block $B_{0}$ and two faithful blocks $B_{7}$ and $B_{8}$. However all simple modules in $B_{7}$ and $B_{8}$ have even dimension, so that by Lemma 2.2(7) none of these blocks can contain endotrivial modules. Whence $T T(3 . \mathrm{McL})=\{[k]\}$.

\subsection{The group Suz.}

The group Suz has a Sylow 2-subgroup $P$ of order $2^{13}$, rank 6 and index 3 in its normaliser $N$, so that $X(N) \cong \mathbb{Z} / 3$. Furthermore $N$ is contained in a maximal subgroup $H$ of Suz of the form $H=2_{-}^{1+6}$. $\mathrm{U}_{4}(2)$ and $|H: N|=135$. Let $1_{10} \in X(N)$ be the 10-th linear character of $N$ (according to the GAP character tables [Bre12] notation), which has order 3. Then

$$
\operatorname{Ind}_{N}^{H}\left(1_{10}\right)=\chi_{8}+\chi_{12}+\chi_{13}+\chi_{18}
$$

where $\chi_{8}, \chi_{12}, \chi_{13}, \chi_{18} \in \operatorname{Irr}(H)$ have degree $15,30,30$ and 60 respectively. Since all four of these characters have classes $2 a$ and $2 b$ in their kernels, the $k H$-Green correspondent $\Gamma_{H}\left(1_{10}\right)$ cannot be endotrivial by Theorem 2.11. Therefore, neither is the $k G$-Green correspondent $\Gamma_{G}\left(1_{10}\right)$ and we obtain $T T(\mathrm{Suz})=\{[k]\}$.

\subsection{The group HN (also denoted $\mathrm{F}_{5}$ or $\mathrm{F}_{5+}$ ).}

The group $G=\mathrm{HN}$ has a Sylow 2-subgroup $P$ of order $2^{14}$ and $G$ has two maximal subgroups which are centralisers of involutions. We gather from $\left[\mathrm{CCN}^{+} 85\right]$ that the centraliser of a central involution has the form $H=2_{+}^{1+8} \cdot\left(\mathfrak{A}_{5} \times \mathfrak{A}_{5}\right)$.2. So $H$ must contain $N_{G}(P)$ and we have $T T(H)=X(H)$ since $H$ has a non-trivial normal 2-subgroup. Now $X(H)=H / P H^{\prime}=1$ because $H^{\prime} \geq\left(\mathfrak{A}_{5} \times \mathfrak{A}_{5}\right)^{\prime}=\mathfrak{A}_{5} \times \mathfrak{A}_{5}$. Therefore, by injectivity of the restriction map $T(G) \rightarrow T(H)$ we conclude that $T T(G)=\{[k]\}$. 


\section{THE TORSION-FREE RANK OF $T F(G)$ IN ODD CHARACTERISTIC AND $p$-RANK $\geq 2$}

Throughout this section, we assume that $p$ is odd. We compute the torsion-free rank $n_{G}$ of $T(G)$, for $G$ a quasi-simple group such that $G / Z(G)$ is sporadic simple and has a noncyclic Sylow $p$-subgroup. Recall from Lemma 2.4 that $n_{G}$ is equal to the number of conjugacy classes of maximal elementary abelian $p$-subgroups of rank 2 if $G$ has $p$-rank 2 , or that number plus one if $G$ has $p$-rank greater than 2 .

5.1. Sporadic groups with $p$-rank 2. First we assume that the $p$-rank is exactly 2. If $P \in \operatorname{Syl}_{p}(G)$, Then a Sylow $p$-subgroup $P$ of $G$ is either elementary abelian of rank 2, or $P$ is an extraspecial $p$-group of order $p^{3}$ and exponent $p$ (see [GLS98]).

Suppose that $P$ is elementary abelian. Then $n_{G}=1$ and $T F(G)=\langle\Omega\rangle \cong \mathbb{Z}$. This occurs in the following cases:

$$
\begin{array}{rl}
\text { characteristic } 3 & \mathrm{M}_{11}, \mathrm{M}_{22}, 2 . \mathrm{M}_{22}, \mathrm{M}_{23}, \mathrm{HS}, 2 . \mathrm{HS} \\
\text { characteristic } 5 & \mathrm{~J}_{2}, 2 . \mathrm{J}_{2}, \mathrm{He}, \mathrm{Suz}, 2 . \mathrm{Suz}, 3 . \mathrm{Suz}, 6 . \mathrm{Suz}, \\
& \mathrm{Fi}_{22}, 2 . \mathrm{Fi}_{22}, 3 . \mathrm{Fi}_{22}, \mathrm{Fi}_{23}, 6 . \mathrm{Fi}_{22}, \mathrm{Fi}_{24}^{\prime},{ }^{2} \mathrm{~F}_{4}(2)^{\prime} \\
\text { characteristic } 7 & \mathrm{Co}_{1}, 2 . \mathrm{Co}_{1}, \mathrm{Th}, \mathrm{B}, 2 . \mathrm{B} \\
\text { characteristic } 11 & \mathrm{M}
\end{array}
$$

Suppose now that $P$ is extraspecial of order $p^{3}$ and exponent $p$, that is $P=p_{+}^{1+2}$ according to Notation 3.1. We use the presentation

$$
P=\left\langle x, y, z \mid x^{p}=y^{p}=z^{p}=[x, z]=[y, z]=1,[x, y]=z\right\rangle
$$

where $[x, y]=x y x^{-1} y^{-1}$. Then $Z(P)=P^{\prime}=\Phi(P)=\langle z\rangle$ has order $p$, where $\Phi(P)$ is the Frattini subgroup. The outer automorphism group of $P$ is isomorphic to $\mathrm{GL}_{2}(p)$ and the matrix $\left(\begin{array}{ll}r^{\prime} & r \\ s^{\prime} & s\end{array}\right)$ with determinant $d$ corresponds to the class of automorphisms sending $x$ to $x^{r^{\prime}} y^{s^{\prime}}, y$ to $x^{r} y^{s}$ and $z$ to $z^{d}$.

Ruiz and Viruel [RV04] have classified the saturated fusion systems on $P$ providing us with the information we need in order to compute $n_{G}$. That is, the number of $G$ conjugacy classes of the $p+1$ elementary abelian $p$-subgroups of rank 2 . We refer the reader to [RV04] for details, and only give the results that apply in our context specifically. We recall that a $p$-subgroup $Q$ is $p$-centric in $G$ if $Z(Q)$ is a Sylow $p$-subgroup of $C_{G}(Q)$, and $Q$ is $p$-radical if $N_{G}(Q) / Q C_{G}(Q)$ has no non-trivial normal $p$-subgroup. Moreover the set of $p$-radical $p$-centric subgroups of $P=p_{+}^{1+2}$ is closed under $G$-conjugation for any finite group $G$, and if this set is empty, then the $p$-fusion is controlled by the normaliser of $P$.

Proposition 5.1. Let $p$ be an odd prime and $G$ be a quasi-simple group such that $G / Z(G)$ is sporadic simple. If the p-rank of $G$ is 2 , then the torsion-free part of $T(G)$ is as given in Table 1.

Proof. By Lemma 2.4, the rank of $T F(G)$ is equal to $n_{G}$. Let $P$ be a Sylow $p$-subgroup of $G$ with $p$-rank 2 .

If $P$ is elementary abelian, then $n_{G}=1$ and $T F(G)=\langle\Omega\rangle \cong \mathbb{Z}$ in the 26 cases listed above. (Note that 26 is a coincidence!) 
TABLE 1. $T F(G)$ for sporadic quasi-simple groups in $p$-rank $2, p>2$

\begin{tabular}{|c|l|l||c|l|l||c|l|l|}
\hline$G$ & $P$ & $T F(G)$ & $G$ & $P$ & $T F(G)$ & $G$ & $P$ & $T F(G)$ \\
\hline $\mathrm{M}_{11}$ & $3^{2}$ & $\mathbb{Z}$ & $\mathrm{McL}$ & $5_{+}^{1+2}$ & $\mathbb{Z}$ & $3 . \mathrm{Fi}_{22}$ & $5^{2}$ & $\mathbb{Z}$ \\
$\mathrm{M}_{12}$ & $3_{+}^{1+2}$ & $\mathbb{Z}^{3}$ & $3 . \mathrm{McL}$ & $5_{+}^{1+2}$ & $\mathbb{Z}$ & $6 . \mathrm{Fi}_{22}$ & $5^{2}$ & $\mathbb{Z}$ \\
$2 . \mathrm{M}_{12}$ & $3_{+}^{1+2}$ & $\mathbb{Z}^{3}$ & $\mathrm{He}$ & $3_{+}^{1+2}$ & $\mathbb{Z}^{2}$ & $\mathrm{Th}$ & $5_{+}^{1+2}$ & $\mathbb{Z}$ \\
$\mathrm{M}_{22}$ & $3^{2}$ & $\mathbb{Z}$ & $\mathrm{He}$ & $5^{2}$ & $\mathbb{Z}$ & $\mathrm{Th}$ & $7^{2}$ & $\mathbb{Z}$ \\
$2 . \mathrm{M}_{22}$ & $3^{2}$ & $\mathbb{Z}$ & $\mathrm{He}$ & $7_{+}^{1+2}$ & $\mathbb{Z}^{3}$ & $\mathrm{Fi}_{23}$ & $5^{2}$ & $\mathbb{Z}$ \\
$3 . \mathrm{M}_{22}$ & $3_{+}^{1+2}$ & $\mathbb{Z}$ & $\mathrm{Ru}$ & $3_{+}^{1+2}$ & $\mathbb{Z}$ & $\mathrm{Co}_{1}$ & $7^{2}$ & $\mathbb{Z}$ \\
$4 . \mathrm{M}_{22}$ & $3^{2}$ & $\mathbb{Z}$ & $2 . \mathrm{Ru}$ & $3_{+}^{1+2}$ & $\mathbb{Z}$ & $2 . \mathrm{Co}_{1}$ & $7^{2}$ & $\mathbb{Z}$ \\
$6 . \mathrm{M}_{22}$ & $3_{+}^{1+2}$ & $\mathbb{Z}$ & $\mathrm{Ru}$ & $5_{+}^{1+2}$ & $\mathbb{Z}^{2}$ & $\mathrm{~J}_{4}$ & $3_{+}^{1+2}$ & $\mathbb{Z}$ \\
$12 . \mathrm{M}_{22}$ & $3_{+}^{1+2}$ & $\mathbb{Z}$ & $2 . \mathrm{Ru}$ & $5_{+}^{1+2}$ & $\mathbb{Z}^{2}$ & $\mathrm{~J}_{4}$ & $11_{+}^{1+2}$ & $\mathbb{Z}$ \\
$\mathrm{J}_{2}$ & $3_{+}^{1+2}$ & $\mathbb{Z}$ & $\mathrm{Suz}$ & $5^{2}$ & $\mathbb{Z}$ & $\mathrm{Fi}_{24}^{\prime}$ & $5^{2}$ & $\mathbb{Z}$ \\
$2 . \mathrm{J}_{2}$ & $3_{+}^{1+2}$ & $\mathbb{Z}$ & $2 . \mathrm{Suz}$ & $5^{2}$ & $\mathbb{Z}$ & $3 . \mathrm{Fi}_{24}^{\prime}$ & $5^{2}$ & $\mathbb{Z}$ \\
$\mathrm{J}_{2}$ & $5^{2}$ & $\mathbb{Z}$ & $3 . \mathrm{Suz}$ & $5^{2}$ & $\mathbb{Z}$ & $\mathrm{Fi}_{24}^{\prime}$ & $7_{+}^{1+2}$ & $\mathbb{Z}^{3}$ \\
$2 . \mathrm{J}_{2}$ & $5^{2}$ & $\mathbb{Z}$ & $6 . \mathrm{Suz}$ & $5^{2}$ & $\mathbb{Z}$ & $3 . \mathrm{Zi}_{24}^{\prime}$ & $7_{+}^{1+2}$ & $\mathbb{Z}^{3}$ \\
$\mathrm{M}_{23}$ & $3^{2}$ & $\mathbb{Z}$ & $\mathrm{O}^{\prime} \mathrm{N}$ & $7_{+}^{1+2}$ & $\mathbb{Z}^{3}$ & $\mathrm{~B}^{2}$ & $7^{2}$ & $\mathbb{Z}$ \\
$\mathrm{HS}$ & $3^{2}$ & $\mathbb{Z}$ & $3 . \mathrm{O}^{\prime} \mathrm{N}$ & $7_{+}^{1+2}$ & $\mathbb{Z}^{3}$ & $2 . \mathrm{B}$ & $7^{2}$ & $\mathbb{Z}$ \\
$2 . \mathrm{HS}$ & $3^{2}$ & $\mathbb{Z}$ & $\mathrm{Co}_{3}$ & $5_{+}^{1+2}$ & $\mathbb{Z}$ & $\mathrm{M}$ & $11^{2}$ & $\mathbb{Z}$ \\
$\mathrm{HS}$ & $5_{+}^{1+2}$ & $\mathbb{Z}^{2}$ & $\mathrm{Co}_{2}$ & $5_{+}^{1+2}$ & $\mathbb{Z}$ & $\mathrm{M}^{2}$ & $13_{+}^{1+2}$ & $\mathbb{Z}^{2}$ \\
$2 . \mathrm{HS}$ & $5_{+}^{1+2}$ & $\mathbb{Z}^{2}$ & $\mathrm{Fi}_{22}$ & $5^{2}$ & $\mathbb{Z}$ & $2 \mathrm{~F}_{4}(2)^{\prime}$ & $3_{+}^{1+2}$ & $\mathbb{Z}^{2}$ \\
$\mathrm{M}_{24}$ & $3_{+}^{1+2}$ & $\mathbb{Z}^{2}$ & $2 . \mathrm{Fi}_{22}$ & $5^{2}$ & $\mathbb{Z}$ & $2 \mathrm{~F}_{4}(2)^{\prime}$ & $5^{2}$ & $\mathbb{Z}$ \\
\hline
\end{tabular}

Now assume $P \cong p_{+}^{1+2}$. So $P$ has $p+1$ elementary abelian $p$-subgroups of rank 2 and $n_{G}$ is the number of $G$-conjugacy classes of such subgroups. We run through the possibilities for $p$ and $G$ according to the classification. The results mentioned below are taken or deduced from [RV04, Table 1.1, Table 1.2, Remark 1.4]. For convenience, let us write $\mathcal{E}$ for the set of the elementary abelian $p$-subgroups of rank 2 of $P$.

The case $p=3$. In this case $|\mathcal{E}|=4$.

If $G \in\left\{\mathrm{Ru}, \mathrm{J}_{4}\right\}$, then all four subgroups in $\mathcal{E}$ are $G$-conjugate, giving $n_{G}=1$.

If $G={ }^{2} \mathrm{~F}_{4}(2)^{\prime}$, then the subgroups in $\mathcal{E}$ split into two conjugacy classes, giving $n_{G}=2$.

If $G \in\left\{\mathrm{M}_{12}, 2 . \mathrm{M}_{12}\right\}$, then fusion in $G$ is the same as in $\mathrm{L}_{3}(3)$ so that $\mathcal{E}$ splits into three conjugacy classes, giving $n_{G}=3$. More precisely, $\{\langle x, z\rangle\}$ and $\{\langle y, z\rangle\}$ are two $G$ conjugacy classes and both subgroups are 3-centric 3-radical. Moreover $N_{G}(P) / P C_{G}(P) \cong$ $2^{2}$ can be realised as the subgroup of diagonal matrices of $\mathrm{GL}_{2}(3)$, so that $\langle x y, z\rangle$ and $\left\langle x y^{2}, z\right\rangle$ become conjugate via the matrix $\left(\begin{array}{ll}1 & 0 \\ 0 & 2\end{array}\right)$.

If $G \in\left\{3 . \mathrm{M}_{22}, 6 . \mathrm{M}_{22}, 12 . \mathrm{M}_{22}\right\}$, then 3 divides the order of the centre of $G$, and because all the subgroups of order 3 of a Sylow 3-subgroup of $\mathrm{M}_{22}$ are conjugate in $\mathrm{M}_{22}$ (cf $\left.\left[\mathrm{CCN}^{+} 85\right]\right)$, the four subgroups of $\mathcal{E}$ in each of the above covers of $\mathrm{M}_{22}$ are $G$-conjugate. Therefore $n_{G}=1$.

If $G \in\left\{\mathrm{M}_{24}, \mathrm{He}\right\}$, then $\mathcal{E}$ splits into two conjugacy classes of size two (cf [RV04, Proof of Lemma 4.8]), one of which is formed by the two 3-centric 3-radical subgroups of $P$ in $G$. So $n_{G}=2$. 
If $G=\mathrm{J}_{2}$, then all four subgroups in $\mathcal{E}$ are $G$-conjugate, giving $n_{G}=1$. Indeed, there is no 3-centric 3-radical subgroup and $N_{G}(P) / P C_{G}(P) \cong 8$ can be realised as the subgroup of $\mathrm{GL}_{2}(3)$ generated by the matrix $\left(\begin{array}{ll}1 & 1 \\ 2 & 1\end{array}\right)$, which sends $x$ to $x y^{2}, y$ to $x y$ and $z$ to $z^{2}$. In consequence all four elements of $\mathcal{E}$ are conjugate.

The case $p=5$. In this case $|\mathcal{E}|=6$.

If $G=$ Th, then all six subgroups in $\mathcal{E}$ are $G$-conjugate, giving $n_{G}=1$.

If $G \in\{\mathrm{Ru}, 2 . \mathrm{Ru}\}$, then $\mathcal{E}$ splits into two conjugacy classes of size two and four (cf [RV04, Proof of Lemma 4.8]). The conjugacy class of size 2 is formed by the 5 -centric 5-radical subgroups of $P$ in $G$, and the other four are conjugate in $N_{G}(P)$. Note indeed that $N_{G}(P) / P C_{G}(P) \cong 4^{2}: 2$.

In all the remaining cases [RV04] show that there are no 5-centric 5-radical subgroups of $P$ in $G$, saying that the $p$-fusion is controlled by $N_{G}(P)$. In other words the $G$ - and $N_{G}(P)$-conjugacy classes of the subgroups in $\mathcal{E}$ coincide, and their number gives us $n_{G}$. We split our analysis into four, according to the structure of $N_{G}(P) / P C_{G}(P)$ as a subgroup of $\mathrm{GL}_{2}(5)$.

If $G=\mathrm{Co}_{3}$, then $N_{G}(P) / P C_{G}(P) \cong D_{48}$ and all six subgroups in $\mathcal{E}$ are $G$-conjugate. Therefore $n_{G}=1$.

If $G=\mathrm{Co}_{2}$, then $N_{G}(P) / P C_{G}(P) \cong 4 . \mathfrak{S}_{4}$ and all six subgroups in $\mathcal{E}$ are $G$-conjugate. Therefore $n_{G}=1$.

If $G \in\{\mathrm{HS}, 2 . \mathrm{HS}\}$, then $N_{G}(P) / P C_{G}(P) \cong D_{16}$ and we calculate that $\mathcal{E}$ splits into two $G$-conjugacy classes. Therefore $n_{G}=2$.

If $G \in\{\mathrm{McL}, 3 . \mathrm{McL}\}$, then $N_{G}(P) / P C_{G}(P) \cong 3: 8$ and all six subgroups in $\mathcal{E}$ are $G$-conjugate. Therefore $n_{G}=1$.

The case $p=7$. In this case $|\mathcal{E}|=8$. We split the analysis into three according to the structure of $N_{G}(P) / P C_{G}(P)$, which is explicitly described as a subgroup of $\mathrm{GL}_{2}(7)$ in [RV04, Proof of Lemma 4.16] allowing us to compute $n_{G}$ by hand. There are five groups to consider.

If $G=$ He, then $N_{G}(P) / P C_{G}(P) \cong \mathfrak{S}_{3} \times 3$ and $\mathcal{E}$ splits into three conjugacy classes: two with three subgroups and one of size two, giving $n_{G}=3$. In this case there are three $G$-conjugate 7-centric 7-radical subgroups.

If $G \in\left\{\mathrm{Fi}_{24}^{\prime}, 3\right.$. $\left.\mathrm{Fi}_{24}^{\prime}\right\}$, then $N_{G}(P) / P C_{G}(P) \cong \mathfrak{S}_{3} \times 6$ and $\mathcal{E}$ splits into three conjugacy classes: two with three subgroups and one of size two, giving $n_{G}=3$. In this case there are six 7-centric 7-radical subgroups split into two $G$-conjugacy classes of size 3 .

If $G \in\left\{\mathrm{O}^{\prime} \mathrm{N}, 3\right.$. O'N $\}$, then $N_{G}(P) / P C_{G}(P) \cong D_{8} \times 3$ and $\mathcal{E}$ splits into three conjugacy classes: two with two subgroups and one of size four, giving $n_{G}=3$. In this case there are four 7-centric 7-radical subgroups split into two $G$-conjugacy classes of size 2.

The case $p=11$. In this case $G=\mathrm{J}_{4}$ and Theorem 3.3 says that $P$ is T.I., which implies $N_{G}(P)=N_{G}(Z(P))$. In this case there are no 11-centric 11-radical subgroups, and so the 11 -fusion is controlled by $N_{G}(P)$. From [Ben81, $\left.\mathrm{CCN}^{+} 85\right]$, we get that $N_{G}(P)=$ $11_{+}^{1+2}:\left(5 \times\left(2 . \mathfrak{S}_{4}\right)\right)$ and $N_{G}(P) / P C_{G}(P) \cong 5 \times\left(2 . \mathfrak{S}_{4}\right)$. Hence the twelve elementary abelian 11-subgroups of $P$ of rank two are $G$-conjugate, and so $n_{G}=1$.

The case $p=13$. In this case $G=\mathrm{M}$ and the fourteen elementary abelian 13subgroups of $P$ of rank two are split into two $G$-conjugacy classes, namely one of size six, consisting of 13-centric 13-radical subgroups, and another of size eight, giving $n_{G}=2$. Here $N_{G}(P) / P C_{G}(P) \cong 3 \times\left(4 . \mathfrak{S}_{4}\right)(\operatorname{cf}[\mathrm{RV04}$, Proof of Lemma 4.18]). 


\subsection{Groups with $p$-rank greater or equal to 3 .}

Proposition 5.2. Let $G$ be a quasi-simple group such that $G / Z(G)$ is sporadic simple and let $p=\operatorname{char}(k)=3$. If $G$ is one of the groups 3.J. $\mathrm{J}_{3}, \mathrm{McL}, 3 . \mathrm{McL}$, Suz, 2.Suz, 3.Suz, O'N, $\mathrm{Co}_{3}, \mathrm{Co}_{2}, \mathrm{Fi}_{22}, 2 . \mathrm{Fi}_{22}, 3 . \mathrm{Fi}_{22}, \mathrm{HN}, \mathrm{Ly}, \mathrm{Th}, \mathrm{Fi}_{23}, \mathrm{Co}_{1}, 2 . \mathrm{Co}_{1}, \mathrm{Fi}_{24}^{\prime}, 3 . \mathrm{Fi}_{24}^{\prime}, \mathrm{B}, 2 . \mathrm{B}$, $\mathrm{M}$, then $T F(G)=\langle\Omega\rangle \cong \mathbb{Z}$.

Proof. In all cases the 3-rank of $G$ is greater or equal to 4, see [GLS98, Table 5.6.1]. Therefore $n_{G}=1$ by Theorem 2.5 and the claim follows from Lemma 2.4.

The remaining cases are the quasi-simple groups $G$ such that $G / Z(G)$ is sporadic simple and $G$ has $p$-rank between 3 and $p$.

For convenience, we introduce a complementary notation taken from [CCN $\left.{ }^{+} 85\right]$. Namely, we write $n X^{e}$, where $n, e$ are positive integers and $X$ a capital letter, to denote an elementary abelian group $n^{e}$ spanned by elements belonging to the $G$-conjugacy class labelled $n X$ in $\left[\mathrm{CCN}^{+} 85\right]$.

Proposition 5.3. Let $p \geq 3$ and $G$ be a quasi-simple group such that $G / Z(G)$ is sporadic simple. If the p-rank of $G$ is greater or equal to 3 and smaller or equal to $p$, then $G$ and TF $(G)$ are as listed in Table 2.

TABLE 2. Sporadic quasi-simple groups with $p$-rank between 3 and $p$.

\begin{tabular}{|c|c|c|c||c|c|c|c||c|c|c|c|}
\hline$G$ & $p$ & $p$-rank & $T F(G)$ & $G$ & $p$ & $p$-rank & $T F(G)$ & $G$ & $p$ & $p$-rank & $T F(G)$ \\
\hline $\mathrm{J}_{3}$ & 3 & 3 & $\mathbb{Z}$ & Ly & 5 & 3 & $\mathbb{Z}$ & $\mathrm{M}$ & 5 & 4 & $\mathbb{Z}$ \\
$3 . \mathrm{O}^{\prime} \mathrm{N}$ & 3 & 3 & $\mathbb{Z}$ & $\mathrm{HN}$ & 5 & 3 & $\mathbb{Z}$ & $\mathrm{M}$ & 7 & 3 & $\mathbb{Z}$ \\
$\mathrm{Co}_{1}$ & 5 & 3 & $\mathbb{Z}^{2}$ & $\mathrm{~B}$ & 5 & 3 & $\mathbb{Z}$ & & & & \\
$2 . \mathrm{Co}_{1}$ & 5 & 3 & $\mathbb{Z}^{2}$ & 2.B & 5 & 3 & $\mathbb{Z}$ & & & & \\
\hline
\end{tabular}

Proof. A Sylow 3-subgroup of $\mathrm{J}_{3}$ has a non-cyclic center and therefore $\mathrm{J}_{3}$ cannot have a maximal elementary abelian 3-subgroup of rank 2. For $3 . \mathrm{O}^{\prime} \mathrm{N}, \mathrm{Co}_{1}$ and $2 . \mathrm{Co}_{1}$, we used MAGMA [BCP97] to calculate the number of conjugacy classes of the elementary abelian $p$-subgroups of rank 2, for the appropriate prime $p$, and then invoked Lemma 2.4. For the remaining cases, we gather information from [CCN ${ }^{+} 85$, GLS98]. Let $P$ be a Sylow $p$-subgroup of $G$. If $G=$ Ly and $p=5$ then $P$ is contained in the normaliser $N_{G}\left(5 A^{3}\right)=$ $5^{3}$. $\mathrm{L}_{3}(5)$ of an elementary abelian subgroup $5 A^{3}$. So $\mathrm{L}_{3}(5)$ acts as automorphism group of a 3-dimensional vector space $\mathbb{F}_{5}^{3}$ and by linear algebra, using Jordan forms, any 5element of $\mathrm{L}_{3}(5)$ must centralise a non-trivial subgroup in $5^{3}$ (i.e. must have a non-trivial eigenspace on $\mathbb{F}_{5}^{3}$ ). Therefore each element of order 5 in $P$ has a centraliser in $P$ of rank at least 3 , saying that $P$ has no maximal elementary abelian subgroup of rank 2. A similar argument applies to B and 2.B, as B has a local subgroup of the form $N_{G}\left(5 B^{3}\right)=5^{3}$. $\mathrm{L}_{3}(5)$. If $G=\mathrm{HN}$ and $p=5$ we look at the normalisers of the elements of order 5 as given in [GLS98]. In the Sylow 5-subgroup of $N_{G}(5 A)=\left(D_{10} \times \mathrm{U}_{3}(5)\right): 2$ each element of order 5 is centralised by a subgroup of 5-rank at least 3 . From $N_{G}(5 B)$ we see that $C_{P}(5 B)$ has rank at least 3 and similarly for $\overline{5 C}$ and $5 E$. Consequently $G$ 
cannot have a maximal elementary abelian subgroup of rank 2. Finally, if $G=\mathrm{M}$ and $p=5$ then $P$ is a Sylow 5 -subgroup of a local subgroup of the form $5_{+}^{1+6} \cdot\left(2 . J_{2}\right) .4$ which has no maximal elementary abelian subgroups of rank 2 and so neither does M. If $p=7$ then there are two conjugacy classes of elements of order 7 . The normalisers have the form $((7.3) \times \mathrm{He}) .2$ and $7_{+}^{1+4} \cdot\left(2 . \mathfrak{A}_{7} \times 3\right) .2$ and none of these has a maximal elementary abelian 7 -subgroup of rank 2 .

\section{The torsion subgroup $T T(G)$ of $T(G)$ in odd Characteristic.}

Let $p$ be an odd prime. We consider in this section groups $G$ with a noncyclic Sylow $p$-subgroup $P$. (If $P$ is cyclic, then we refer to Section 7.) By Lemma 2.8, the torsion subgroup $T T(G)$ identifies with a subgroup of $X\left(N_{G}(P)\right)$ via restriction and consists precisely of the $k G$-Green correspondents of the 1-dimensional $k N_{G}(P)$-modules that are endotrivial, i.e. the trivial source endotrivial modules. Recall that for a group $G$ we write $X(G)$ for the group of isomorphism classes of 1-dimensional $k G$-modules, and it is isomorphic to the $p^{\prime}$-group $G / G^{\prime} P$ where $G^{\prime}=[G, G]$ is the commutator subgroup of $G$.

Simple endotrivial modules for covering groups of sporadic groups have been determined in [LMS13, $\S 7$ ], where the authors prove that many of them are trivial source modules, hence elements of $T(G)$. We now continue our analysis of $T T(G)$ along similar lines, using Green correspondence and induction and restriction of characters.

In the rest of this section we use the following notation. If $G$ is a given sporadic group and $\ell . G$ an $\ell$-fold cover of $G$, we write $P$ and $\widetilde{P}$ for a Sylow $p$-subgroup of $G$ and of $\ell . G$ respectively, and set $N=N_{G}(P)$ and $\widetilde{N}=N_{\ell . G}(\widetilde{P})$. Also, for a subgroup $H$ of $G$ containing $N$ and for an indecomposable $k N$-module $V$, then $\Gamma_{H}(V)$ denotes the $k H$-Green correspondent of $V$. Similarly for $\ell . G$.

We use the structure of the Sylow normalisers and maximal subgroups as given in $\left[\mathrm{CCN}^{+} 85, \mathrm{GL} 83, \mathrm{GLS} 98\right]$ and the GAP character table librairies [Bre12]. These are especially useful in determining $X(N)$. In our computations we denote by $\chi$ ordinary irreducible characters of a group. We index the ordinary irreducible charcters and the blocks according to the GAP character table libraries [Bre12], with the exception that we denote the principal block by $B_{0}$ instead of $B_{1}$. Accordingly we denote by $e_{i}$ the central primitive idempotent corresponding to the block $B_{i}$. We denote by $1_{H} \in \operatorname{Irr}(H)$ the trivial character of a group $H$ and $1_{i} \in X(H)$ denotes a non-trivial 1-dimensional $k H$-module. We also identify these with the corresponding ordinary and Brauer characters, as well as the corresponding simple $k G$-module and its class in $X(H)$.

Throughout we use the dimensional criterion for trivial source endotrivial modules of Lemma 2.2(7), the possible character values for characters of endotrivial modules given by Theorem 2.10 and Theorem 2.11, and the possible values of characters of trivial source modules given by Lemma 2.12. We split the cases according to the values of $p$ and the arguments we used to obtain $T T(G)$.

First, as for the 2-fold covers in characteristic 2, the 3 -fold covers in characteristic 3 cannot have non-trivial torsion endotrivial modules.

Lemma 6.1. Let $p=3$ and let $G$ be one of the covering groups $3 . \mathrm{M}_{22}, 6 . \mathrm{M}_{22}, 12 . \mathrm{M}_{22}$, 3.J $\mathrm{J}_{3}$ 3.McL, 3.Suz, 6.Suz, 3.O'N, 3. $\mathrm{Fi}_{22}, 6 . F i_{22}$, 3. $\mathrm{Fi}_{24}^{\prime}$. Then $T T(G)=\{[k]\}$. 
Proof. In all cases $G$ has a non-trivial normal 3-subgroup so that $T T(G)=\{[k]\}$ by Lemma 3.1(2).

Next we treat covering groups $G$ such that $p \nmid|Z(G)|$.

Lemma 6.2. Assume $p=3$ and $G$ is one of the covering groups $2 . \mathrm{M}_{12}, 4 . \mathrm{M}_{22}, 2 . \mathrm{J}_{2}$, 2. HS, 2.Suz, 2. $\mathrm{Fi}_{22}, 2 . \mathrm{Co}_{1}, 2 . \mathrm{B}$, or $p=5$ and $G$ is one of the covering groups $2 . \mathrm{J}_{2}, 2 . \mathrm{HS}$, 2.Ru, 2.Suz, 3.Suz, 6.Suz, 2. $\mathrm{Co}_{1}$, 3. $\mathrm{Fi}_{24}^{\prime}, 2 . \mathrm{B}$, or $p=7$ and $G$ is one of the covering groups 2. $\mathrm{Co}_{1}, 3 . \mathrm{Fi}_{24}^{\prime}, 2$.B. Then $G$ has no faithful endotrivial module. Furthermore in all cases $T(G) \cong T(G / Z(G))$ via inflation, except if $G=4 \cdot \mathrm{M}_{22}$ in which case $T(G) \cong T\left(2 \cdot \mathrm{M}_{22}\right)$.

Proof. Since $p \nmid|Z(G)|$ faithful endotrivial modules must afford characters in faithful blocks. The proof is similar to that of Lemma 4.3. Except for 2.HS in characteristic 3 , the GAP character table libraries [Bre12] show in all cases that $G$ has a $p$-singular conjugacy class $C$ such that for all faithful characters $\psi \in \operatorname{Irr}(G)$ we have $\psi(c) \in m \mathbb{Z}$ with $m \in \mathbb{Z} \backslash\{ \pm 1\}, c \in C$. Hence a contradiction with the possible values of characters of endotrivial modules given by Theorem 2.10. More accurately, we can choose $m$ and $C$ as follows. For $p=3$ take $m=0$ if $G$ is one of $2 . \mathrm{M}_{12}, 4 . \mathrm{M}_{22}, 2 . \mathrm{J}_{2}, 2 . \mathrm{Suz}_{2} 2 . \mathrm{Fi}_{22}, 2 . \mathrm{Co}_{1}$, or $2 . \mathrm{B}$, and for $C$ choose $12 a, 6 b, 12 a, 12 a, 12 a, 12 a, 6 c$ respectively. For $p=3$ and $G=2 . \mathrm{M}_{12}$ take $(m, C)=(2,6 c)$. If $p=5$ take $m=0$ if $G$ is one of 2. $\mathrm{J}_{2}, 2 . \mathrm{HS}, 2$.Suz, 3.Suz, 6.Suz, 2. $\mathrm{Co}_{1}$, or 2.B and for $C$ choose 20a, 20a, 20a, 15e, 15e, 20a, $20 b$ respectively. For $p=5$, if $G=2$.Ru take $(m, C)=(5,5 b)$, and if $G=3$.Fi ${ }_{24}^{\prime}$ take $(m, C)=(5,15 c)$. If $p=7$ take $(m, C)=(2,7 a)$ for $G=2 . \mathrm{Co}_{1}$, take $(m, C)=(3,7 a)$ for $G=3 . \mathrm{Fi}_{24}^{\prime}$, and take $(m, C)=(0,14 b)$ for $G=2$.B. Finally if $G=2$.HS and $p=3$, then all faithful characters take value in $3 i \mathbb{Z}$ on class $12 a$, hence the result. (Notation for conjugacy classes is that of GAP.) The claim on $T(G)$ is straightforward from Lemma 3.2.

We now go through the list of sporadic simple groups by increasing order, including non-trivial covers not treated by Lemmas 6.1 and 6.2 above, and compute the torsion subgroup $T T(G)$ for as many groups $G$ and characteristics $p$ as possible. In the cases when we do not obtain the full structure of $T T(G)$ we give bounds instead.

Let us point out that the results for the groups He and Suz in characteristic 5, hereafter mentioned as 'MAGMA computation', partly use a method recently developed by Paul Balmer [Bal13] and a subsequent algorithm by Carlson (private communication). Balmer's method determines the kernel of the restriction $T T(G) \rightarrow T T(H)$ to a subgroup $H$ of $G$ by showing that this kernel is isomorphic to the set of the so-called $H$-weak homomorphisms. In particular, for $H=P$ the set of weak $P$-homomorphisms is isomorphic to the subgroup of $T T(G)$ spanned by the trivial source endotrivial modules. We omit here the technicalities, to avoid further deviation from our present scope, referring the reader to [Bal13]. The main drawback of this method is that it cannot always be used in practice, and when it does, then it often relies on tedious computer calculations. Both authors are thankful to Jon Carlson with his help in implementing an algorithm on MAGMA and running it on specific groups.

6.1. The group $G=\mathrm{M}_{11}$ in characteristic 3. By Theorem 3.3, the group $G$ has an abelian T.I. Sylow 3 -subgroup $P \cong 3^{2}$ and $N \cong 3^{2}: S D_{16}$. Thus $X(N) \cong S D_{16} /\left(S D_{16}\right)^{\prime} \cong$ $(\mathbb{Z} / 2)^{2}$. It follows from Lemma $2.8(3),(4)$ and Proposition 5.1 that $T T(G) \cong X(N) \cong$ $(\mathbb{Z} / 2)^{2}$. 
The indecomposable modules representing the classes in $T T(G)$ are the $k G$-Green correspondents of the modules in $X(N)$. Their Loewy and socle series, as well as the characters they afford, are described in [KW99a, (4.3)]. Their dimensions are 1, 55, 55 and 10.

6.2. The group $G=\mathrm{M}_{12}$ and its double cover in characteristic 3 . In this case $P=p_{+}^{1+2}$ and we have a chain of subgroups

$$
P \leq N \leq N_{2} \leq \mathrm{M}_{12} \quad \text { where } N \cong 3_{+}^{1+2}: 2^{2} \text { and } N_{2} \cong 3^{2}: \mathrm{GL}_{2}(3) .
$$

So $X(N) \cong(\mathbb{Z} / 2)^{2}$ and $X\left(N_{2}\right) \cong \mathbb{Z} / 2$. Since $\left|N_{2}: N\right|=4$, induction from $k N$ to $k N_{2}$ of a 1-dimensional $k N$-module is a module of dimension 4 . Hence the $k N_{2^{-}}$ Green correspondents $\Gamma_{N_{2}}\left(1_{N}\right), \Gamma_{N_{2}}\left(1_{1}\right), \Gamma_{N_{2}}\left(1_{2}\right), \Gamma_{N_{2}}\left(1_{3}\right)$ of the four 1-dimensional modules $1_{N}, 1_{1}, 1_{2}, 1_{3} \in X(N)$ have dimension $1,1,4$ and 4 , respectively, because $N_{2}$ has exactly two non-isomorphic 1-dimensional modules. In particular we conclude that $\Gamma_{N_{2}}\left(1_{2}\right)$, $\Gamma_{N_{2}}\left(1_{3}\right)$ are not endotrivial, for $4 \not \equiv 1(\bmod |P|)$. A fortiori $\Gamma_{G}\left(1_{2}\right), \Gamma_{G}\left(1_{3}\right)$ are not endotrivial either. It remains to prove that $\Gamma_{G}\left(1_{2}\right) \notin T T(G)$. Note that the principal block $B_{0}$ is the unique block of $k G$ with full defect. The Loewy and socle series of $\Gamma_{G}\left(1_{1}\right)$ have been computed in [KW99b, 6.1]. In particular, if $\psi_{1}$ denotes the linear character corresponding to $\Gamma_{N_{2}}\left(1_{1}\right)$, then we see that $e_{0} \cdot \operatorname{Ind}_{N_{2}}^{G}\left(\psi_{1}\right)$ yields that $\Gamma_{G}\left(1_{1}\right)$ is the trivial source module affording the character $\chi_{9}+\chi_{13}$ of degree $175 \equiv 13(\bmod |P|) \not \equiv 1(\bmod |P|)$. Whence $\Gamma_{G}\left(1_{1}\right) \notin T T(G)$ and $T T(G)=\{[k]\}$.

6.3. The group $G=\mathrm{M}_{22}$ and its covers in characteristic 3. A Sylow 3-subgroup $P$ of $G$ is elementary abelian of order 9 and $N \cong 3^{2}: Q_{8}$. So $X(N) \cong(\mathbb{Z} / 2)^{2}$. In order to obtain $T T(G)$, we first consider the group $2 . G=2 . M_{22}$. A Sylow 3 -subgroup $\widetilde{P}$ of $2 . G$ is elementary abelian of order 9 and $X(\widetilde{N}) \cong \mathbb{Z} / 2 \oplus \mathbb{Z} / 4$. We gather from [LMS13, Thm. 7.1] and [LM14, Sec. 2] that 2.G has six simple trivial source endotrivial modules. Namely, the trivial module $k$, a self-dual module $S_{55}$ of dimension 55 affording the character $\chi_{5} \in \operatorname{Irr}(2 . G)$, and four faithful modules: $S_{10}, S_{10}^{\prime}$, both of dimension 10, affording the characters $\chi_{13}, \chi_{14} \in \operatorname{Irr}(2 . G)$, and $S_{154},\left(S_{154}\right)^{*}$ both of dimension 154, affording the characters $\chi_{19}, \chi_{20} \in \operatorname{Irr}(2 . G)$. It follows directly that $T T(2 . G) \cong X(\widetilde{N}) \cong \mathbb{Z} / 2 \oplus \mathbb{Z} / 4$. Furthermore, by Lemma 3.2(2), Green correspondence preserves the faithfulness of blocks, hence $T T(G) \cong(\mathbb{Z} / 2)^{2}$. The group $T\left(4 \cdot \mathrm{M}_{22}\right)$ is isomorphic to $T\left(2 \cdot \mathrm{M}_{22}\right)$ via inflation by Lemma 6.2. However, notice that $T T\left(4 . \mathrm{M}_{22}\right) \nsucceq X(\widetilde{N}) \cong \mathbb{Z} / 2 \oplus \mathbb{Z} / 8$ in this case. Finally the groups $T T\left(3 . \mathrm{M}_{22}\right), T T\left(6 . \mathrm{M}_{22}\right)$ and $T T\left(12 . \mathrm{M}_{22}\right)$ are all trivial by Lemma $3.1(2)$.

\subsection{The group $G=\mathrm{J}_{2}$.}

Characteristic 3 . In this case $P \cong 3_{+}^{1+2}, N \cong 3_{+}^{1+2}: 8, X(N) \cong \mathbb{Z} / 8$ and $B_{0}(k G)$ is the unique 3 -block with full defect. We pick $1_{4}$ to be a character of order 4 of $X(N)$, then $e_{0} \cdot \operatorname{Ind}_{N}^{G}\left(1_{4}\right)=\chi_{12}+\chi_{13}+2 \chi_{21}$. By the criteria on degrees and character values $\Gamma_{G}\left(1_{4}\right)$ cannot be endotrivial. It follows that $T T(G)$ can at most be cyclic of order 2 . Let $1_{7}$ denote the linear character of order 2 of $N$. We have $e_{0} \cdot \operatorname{Ind}_{N}^{G}\left(1_{7}\right)=\chi_{4}+\chi_{5}+\chi_{12}+$ $2 \chi_{13}+\chi_{16}+\chi_{17}+\chi_{20}+2 \chi_{21}$. Again using the possible degrees and values of trivial source characters given by Lemma 2.12 it is easy to see that $\Gamma_{G}\left(1_{7}\right)$ must afford the character $\chi_{4}+\chi_{5}+\chi_{13}$, which takes value 1 on all the non-trivial 5 -elements and is thus endotrivial Theorem 2.11. We conclude that $T T(G) \cong \mathbb{Z} / 2$. 
Characteristic 5. In this case $P$ is elementary abelian of rank 2 and $N \cong 5^{2}: D_{12}$ so that $X(N) \cong(\mathbb{Z} / 2)^{2}$. The principal 5-block of $k G$ is the unique block with full defect. Let $X(N)=\left\{1_{N}, 1_{1}, 1_{3}, 1_{4}\right\}$. Inducing the non-trivial characters in $X(N)$ we get the following. First $e_{0} \cdot \operatorname{Ind}_{N}^{G}\left(1_{1}\right)=\chi_{14}+\chi_{15}+\chi_{19}$, so that by the degree and character value criteria we obtain that $\Gamma_{G}\left(1_{1}\right)$ affords $e_{0} \cdot \operatorname{Ind}_{N}^{G}\left(1_{1}\right)$, which does not take value 1 on all non-trivial 5-elements. Second $e_{0} \cdot \operatorname{Ind}_{N}^{G}\left(1_{3}\right)=\chi_{14}+\chi_{15}+\chi_{16}+\chi_{17}$. Again by the degree criteria, we must have that $\Gamma_{G}\left(1_{3}\right)$ affords $e_{0} \cdot \operatorname{Ind}_{N}^{G}\left(1_{3}\right)$, as it takes value 1 on all the non-trivial 5-elements. By Theorem 2.11, $\Gamma_{G}\left(1_{3}\right)$ is endotrivial whereas $\Gamma_{G}\left(1_{1}\right)$ is not. Whence $T T(G) \cong \mathbb{Z} / 2$.

6.5. The group $G=\mathrm{M}_{23}$ in characteristic 3. In this case $P$ is elementary abelian of order 9 and $N \cong 3^{2}: Q_{8} .2$. It follows that $X(N) \cong(\mathbb{Z} / 2)^{2}$.

By [LMS13, Thm. 7.1], $\mathrm{M}_{23}$ has a simple self-dual endotrivial module $S_{253}$ of dimension 253. Hence $\mathbb{Z} / 2 \cong\left\langle\left[S_{253}\right]\right\rangle \leq T T(G)$.

Now, the natural permutation $k G$-module on 23 points has a 22-dimensional composition factor $S_{22}$, which must be a direct summand. Therefore $S_{22}$ is a trivial source module with vertex $P$ and its $k N$-Green correspondent is simple (see [DK09, §3.7]). Thus must have dimension at most 2 . Since $22 \equiv 1(\bmod 3)$, we conclude that $S_{22}$ is the $k G$-Green correspondent of a 1-dimensional $k N$-module. But as $22 \not \equiv 1(\bmod |P|)$, the module $S_{22}$ is not endotrivial. Consequently $T T(G) \cong \mathbb{Z} / 2$.

6.6. The group $G={ }^{2} \mathrm{~F}_{4}(2)^{\prime}$.

Characteristic 3 . In this case $P$ is extraspecial of order 27 and exponent 3 and $N \cong$ $3_{+}^{1+2}: D_{8}$. Hence $X(N) \cong(\mathbb{Z} / 2)^{2}$ and we denote $1_{N}, 1_{1}, 1_{2}, 1_{3}$ the four non-isomorphic 1-dimensional $k N$-modules.

The group $G$ has a maximal subgroup $H \cong \mathrm{L}_{3}(3) .2$ of index 1600 which contains $N$ and $X(H)=\left\{1_{H}, 1_{a}\right\}$ has order 2. We may assume that $\Gamma_{H}\left(1_{N}\right)=1_{H}$ and $\Gamma_{H}\left(1_{1}\right)=1_{a}$. Induction from $N$ to $H$ yields

$$
\operatorname{Ind}_{N}^{H}\left(1_{2}\right)=\chi_{5}+\chi_{13} \quad \text { and } \quad \operatorname{Ind}_{N}^{H}\left(1_{3}\right)=\chi_{6}+\chi_{14} .
$$

Since $\chi_{5}(1)=\chi_{6}(1)=13$ and $\chi_{13}(1)=\chi_{14}(1)=39$, and $13,39,13+39 \not \equiv 1(\bmod |P|)$, the $k H$-Green correspondents $\Gamma_{H}\left(1_{2}\right)$ and $\Gamma_{H}\left(1_{3}\right)$ cannot be endotrivial. Similarly, $\operatorname{Ind}_{H}^{G}\left(1_{a}\right)=$ $\chi_{7}+\chi_{19}$ where $\chi_{7}(1)=300 \not \equiv 1(\bmod |P|), \chi_{19}(1)=1300 \not \equiv 1(\bmod |P|)$ and $\left(\chi_{7}+\right.$ $\left.\chi_{19}\right)(1) \not \equiv 1(\bmod |P|)$ so that the $k G$-Green correspondent of $1_{1}$ is not endotrivial. Therefore $T T(H) \cong \mathbb{Z} / 2$ and $T T(G) \cong\{[k]\}$.

Characteristic 5. In this case $P$ is elementary abelian of rank 2 and $N \cong\left(5^{2}\right):\left(4 . \mathfrak{A}_{4}\right)$. Theorem 3.3 says that $P$ is a T.I. set, so that by Lemma $2.8(4),(5)$ we get that $T T(G) \cong$ $T T(N) \cong X(N)$ because $N$ is strongly $p$-embedded in $G$. Finally we compute that $X(N) \cong \mathbb{Z} / 6$.

\subsection{The group $G=\mathrm{HS}$.}

Characteristic 3 . In this case $P \cong 3^{2}$ and $N \cong 2 \times\left(3^{2} . S D_{16}\right)$. So $X(N) \cong(\mathbb{Z} / 2)^{3}$. By [LMS13, Thm. 7.1], $G$ has three simple endotrivial modules $S_{4}, S_{5}, S_{6}$ all of dimension 154 , and affording the characters $\chi_{4}, \chi_{5}, \chi_{6}$ respectively. From [LM14, Sec.2] we have $T T(G)=\left\langle\left[S_{4}\right],\left[S_{5}\right],\left[S_{6}\right]\right\rangle \cong(\mathbb{Z} / 2)^{2}$. Alternatively, a MAGMA computation (not involving Balmer's method described above) shows that there exists a $k N$-module $1_{a} \in X(N)$ 
such that its $k G$-Green correspondent is of dimension $175 \not \equiv 1(\bmod 9)$, so that $T T(G)$ certainly cannot have order 8 .

Characteristic 5. In this case $P \cong 5_{+}^{1+2}$, and $N \cong 5_{+}^{1+2}:(8.2)$. Also $G$ has a maximal subgroup $H^{*} \cong \mathrm{U}_{3}(5) .2$ containing $N$. We get $X(N) \cong \mathbb{Z} / 2 \oplus \mathbb{Z} / 4, X\left(H^{*}\right) \cong$ $\mathbb{Z} / 2$. We note that $N$ is strongly 5 -embedded in $H^{*}$, so that $T T\left(H^{*}\right) \cong \mathbb{Z} / 2 \oplus \mathbb{Z} / 4$ by Lemma 2.8. Now if $1_{a} \in X\left(H^{*}\right)$ is non-trivial, we have $e_{0} \cdot \operatorname{Ind}_{H^{*}}^{G}\left(1_{a}\right)=\chi_{2}+\chi_{5}$, where $\chi_{2}(1)=22, \chi_{5}(1)=154$. Therefore $\Gamma_{G}\left(1_{1}\right)=e_{0} \cdot \operatorname{Ind}_{H^{*}}^{G}\left(1_{a}\right)$ and affords $\chi_{2}+\chi_{5}$, but is not endotrivial since its dimension is $176 \not \equiv 1(\bmod |P|)$. From the group structure of $X(N)$, we see that $T T(G) \leq \mathbb{Z} / 4$. Now the linear character $1_{5} \in X(N)$ has order 4 and

$$
e_{0} \cdot \operatorname{Ind}_{N}^{G}\left(1_{5}\right)=\chi_{8}+\chi_{9}+\chi_{10}+\chi_{16}+\chi_{17}+2 \cdot \chi_{22} .
$$

(Note that $B_{0}$ is the unique 5-block with full defect.) It follows from the possible values of trivial source modules given by Lemma 2.12 and the 5 -decomposition matrix of $G$, that $\Gamma_{G}\left(1_{5}\right)$, affords either the character $\chi_{8}+\chi_{10}$ or the character $\chi_{8}+\chi_{22}$. In both cases we obtain that $\Gamma_{G}\left(1_{5}\right)$ is endotrivial by Theorem 2.11 and we conclude that $T T(G) \cong \mathbb{Z} / 4$.

6.8. The group $G=\mathrm{J}_{3}$ in characteristic 3 . In this case $P$ has order $3^{5}$ and $N \cong$ $3^{2} \cdot\left(3^{1+2}\right): 8$ so that $X(N) \cong \mathbb{Z} / 8$ (note that $N$ is maximal in $G$ ). The linear character $1_{4}$ has order 4 in $X(N)$ and

$$
e_{0} \cdot \operatorname{Ind}_{N}^{G}\left(1_{4}\right)=2 \chi_{10}+2 \chi_{13}+\chi_{14}+\chi_{15}+\chi_{16}+\chi_{17}+\chi_{18}+\chi_{19} .
$$

It follows from the character table of $J_{3}$ that if $\operatorname{dim}_{k} \Gamma_{G}\left(1_{4}\right) \equiv 1(\bmod |P|)$, then its character cannot take value 1 on all non-trivial 3 -elements. Hence, by Theorem 2.11, $\Gamma_{G}\left(1_{4}\right)$ is not endotrivial. This proves that $T T(G) \leq \mathbb{Z} / 2$.

6.9. The group $G=\mathrm{M}_{24}$ in characteristic 3 . We show that $T T(G)=\{[k]\}$.

In this case $P$ is extraspecial of order 27 and exponent 3 and $N \cong 3_{+}^{1+2}: D_{8}$ where $D_{8}$ is a dihedral group of order 8 . From $\left[\mathrm{CCN}^{+} 85\right]$, we see that $G$ has a maximal subgroup $H \cong M_{12}: 2$ containing $N$. Therefore $X(H) \cong \mathbb{Z} / 2$ and $X(N) \cong(\mathbb{Z} / 2)^{2}$. We denote by $1_{H}, 1_{a}$ the elements of $X(H)$ and by $1_{N}, 1_{1}, 1_{2}$ and $1_{3}$ the elements of $X(N)$. By Green correspondence, we may assume that $\Gamma_{H}\left(1_{N}\right)=1_{H}$ and $\Gamma_{H}\left(1_{1}\right)=1_{a}$. Inducing the character $1_{3}$ to $H$ gives

$$
e_{0} \cdot \operatorname{Ind}_{N}^{H}\left(1_{3}\right)=\chi_{12}+\chi_{15}+\chi_{16}+\chi_{17}+\chi_{21} .
$$

(Note that the principal block is the unique 3-block of $k H$ with full defect). Using Theorem 2.11 and Lemma 2.12 it is easy to check that for degree reasons, or character values on $p$-elements, $\Gamma_{H}\left(1_{3}\right)$ cannot be endotrivial. Consequently $T T(H)=X(H) \cong$ $\mathbb{Z} / 2$.

Finally $1_{a} \uparrow_{H}^{G}=\chi_{2}+\chi_{17}$, where $\chi_{2}(1)=23$ and $\chi_{17}(1)=1265 \equiv 23(\bmod |P|)$, so that again for degree reasons the $k G$-Green correspondent of $1_{a}$ is not endotrivial either. The claim follows.

\subsection{The group $G=\mathrm{McL}$ and its 3 -fold cover.}

Characteristic 3 . In this case $P$ has order $3^{6}$ and $N \cong 3^{4}:\left(3^{2}: Q_{8}\right)$ so that $X(N) \cong$ $(\mathbb{Z} / 2)^{2}$. In addition $G$ has a subgroup $H \cong 3^{1+4}:\left(2 . \mathfrak{S}_{5}\right)$. Since $|H: N|=10<|P|$, we have $T T(H) \cong X(H) \cong \mathbb{Z} / 2$. So let $1_{a}$ be the non-trivial linear character of $H$. Then $\operatorname{Ind}_{H}^{G}\left(1_{a}\right)=\chi_{3}+\chi_{15}+\chi_{20}$. Now as $\chi_{3}, \chi_{15}, \chi_{20}$ all take a value strictly greater 
than 1 on conjugacy class $3 a, \Gamma_{G}\left(1_{a}\right)$ cannot be endotrivial by Theorem 2.11 . Whence $T T(G)=\{[k]\}$, whereas the group $T T(3 . \mathrm{McL})$ is trivial by Lemma 3.1(2).

Characteristic 5. In this case $P=5_{+}^{1+2}$ and $N \cong 5_{+}^{1+2}: 3: 8$ is a maximal subgroup of $G$. Moreover $P$ is a T.I. set by Theorem 3.3 and therefore by Lemma 2.8(4),(5) we get that $T T(G) \cong X(N) \cong \mathbb{Z} / 8$. The situation is similar for the 3 -fold cover $3 . \mathrm{McL}$, i.e. $\widetilde{P} \cong 5_{+}^{1+2}$ and is a T.I. set as well. The normaliser $\widetilde{N}$ is an extension of degree 3 of $N$ and $X(\widetilde{N}) \cong \mathbb{Z} / 24$. Whence $T T(3 . \mathrm{McL}) \cong X(\widetilde{N}) \cong \mathbb{Z} / 24$.

6.11. The group $G=\mathrm{He}$.

Characteristic 3 . In this case $P \cong 3_{+}^{1+2}$ and $N \cong 3_{+}^{1+2}: D_{8}$ so that $X(N) \cong$ $(\mathbb{Z} / 2)^{2}$. The group $G$ has a maximal subgroup $H \cong 3 \cdot A_{7} .2$ containg $N$. It follows from Lemma $2.8(3)$ that $T T(H) \cong X(H)$, where $X(H)=\left\{1_{H}, 1_{2}\right\} \cong \mathbb{Z} / 2$. Hence $T T(G) \leq$ $T T(H) \cong \mathbb{Z} / 2$ via restriction and we only need to check whether $\Gamma_{G}\left(1_{2}\right)$ is endotrivial. $\operatorname{But}_{\operatorname{Ind}_{H}^{G}}^{G}\left(1_{2}\right)=\chi_{14}+\chi_{15}+\chi_{22}+2 \cdot \chi_{29}+\chi_{30}+\chi_{31}$ so that it follows easily from the values of $\chi_{14}, \chi_{15}, \chi_{22}, \chi_{29}, \chi_{30}, \chi_{31} \in \operatorname{Irr}(G)$ that the character afforded by $\Gamma_{G}\left(1_{2}\right)$ cannot take value 1 on conjugacy class $3 b$. Whence $T T(G) \cong\{[k]\}$.

Characteristic 5. In this case $P$ is elementary abelian of order 25 and $N \cong 5^{2}:\left(4 . \mathfrak{S}_{4}\right)$. So $X(N) \cong \mathbb{Z} / 6$. By [LMS13, Thm. 7.1], $G$ has two simple trivial source endotrivial modules $S_{51}$ and $S_{51}^{*}$, both of dimension 51, affording the characters $\chi_{2}, \chi_{3} \in \operatorname{Irr}(G)$. Now $\chi_{1} \downarrow_{N}$ and $\chi_{2} \downarrow_{N}$ have exactly two linear constituents $\chi_{4}$ and $\chi_{6}$, both of which have order 3 in $X(N)$. Therefore $S_{51}, S_{51}^{*}$ have order 3 in $T T(G)$, since they are the $k G$-Green correspondents of 1-dimensional modules of order 3 in $X(N)$, showing that $T T(G)$ has order at least 3 . Finally a MAGMA computation shows that $T T(G) \cong \mathbb{Z} / 3$.

6.12. The group $G=\mathrm{Ru}$ and its cover in characteristic 3 . In this case $P$ is extraspecial of order 27 and exponent 3 and $N \cong 3_{+}^{1+2}: S D(16)$. So $X(N) \cong(\mathbb{Z} / 2)^{2}$. For $2 . G$, we have $\widetilde{P} \cong P$ and $X(\widetilde{N}) \cong \mathbb{Z} / 2 \oplus \mathbb{Z} / 4$.

By [LMS13, Thm. 7.1], $G$ has a self-dual simple trivial source endotrivial module $S_{406}$ of dimension 406, affording the character $\chi_{4} \in \operatorname{Irr}(G)$. Therefore $\mathbb{Z} / 2 \cong\left\langle\left[S_{406}\right]\right\rangle \leq T T(G)$. Again by [LMS13, Thm. 7.1], 2.G has two faithful simple endotrivial modules $S_{28}$ and $S_{28}^{*}$, both of dimension 28, affording the characters $\chi_{37}, \chi_{38} \in \operatorname{Irr}(2 . G)$. Moreover $\chi_{37} \otimes \chi_{37}=$ $\chi_{2}+\chi_{4}$ where $\chi_{2}$ has defect zero. Reduction modulo 3 yields $S_{28} \otimes S_{28} \cong S_{406} \oplus$ (proj) saying that $S_{28}$ is a trivial source module of order 4 in $T T(\widetilde{G})$. Therefore $\mathbb{Z} / 4 \cong\left\langle\left[S_{28}\right]\right\rangle \leq$ $T T(2 . G)$.

Now $G$ has a maximal subgroup $H \cong\left(2^{6}: \mathrm{U}_{3}(3)\right): 2$ containing $N$ with $X(H) \cong \mathbb{Z} / 2$. If $1_{2} \in X(H)$ denotes the non-trivial character of $H$, then $e_{0} \cdot \operatorname{Ind}_{H}^{G}\left(1_{2}\right)=\chi_{9}+\chi_{25}$. For degree reasons $\Gamma_{G}\left(1_{1}\right)$ affords $\chi_{9}+\chi_{25} \in \mathbb{Z} \operatorname{Irr}(G)$, but $\chi_{9}+\chi_{25}$ takes value 31 on class $3 a$, so that $\Gamma_{G}\left(1_{1}\right)$ is not endotrivial by Theorem 2.11. This yields $T T(H) \cong T T(G) \cong \mathbb{Z} / 2$ and $T T(2 . G) \cong \mathbb{Z} / 4$.

\subsection{The group $G=$ Suz.}

Characteristic 3 . In this case $P \cong 3^{5}: 3^{2}, N \cong 3^{5}:\left(3^{2}: S D_{16}\right)$ and $X(N) \cong(\mathbb{Z} / 2)^{2}$. Moreover $G$ has a subgroup $H \cong 3^{5}: M_{11}$ containing $N$, so that $T T(G) \leq T T(H)$ via restriction. Inducing the three non-trivial linear characters $1_{1}, 1_{2}, 1_{4}$ of $N$ to $H$ we get:

$$
e_{0} \cdot \operatorname{Ind}_{N}^{H}\left(1_{1}\right)=\chi_{2}+\chi_{9}, \quad e_{0} \cdot \operatorname{Ind}_{N}^{H}\left(1_{2}\right)=\chi_{5}+\chi_{8}, \quad e_{0} \cdot \operatorname{Ind}_{N}^{H}\left(1_{4}\right)=\chi_{10}
$$


(Note that $H$ has a unique block of full defect.) Since $\chi_{2}, \chi_{5}, \chi_{8}, \chi_{9}, \chi_{10} \in \operatorname{Irr}(H)$ all have degree strictly greater than 1 and class $3 a$ in their kernel, it follows that the $k H$-Green correspondents $\Gamma_{H}\left(1_{1}\right), \Gamma_{H}\left(1_{2}\right), \Gamma_{H}\left(1_{4}\right)$ cannot be endotrivial by Theorem 2.11. A fortiori $T T(G)=T T(H)=\{[k]\}$.

Characteristic 5. By [LMS13, Thm. 7.1] the character $\chi_{5} \in \operatorname{Irr}($ Suz) reduces modulo 5 to a self-dual endotrivial $k$ Suz-module $S_{1001}$ of dimension 1001 . Therefore $\mathbb{Z} / 2 \leq$ $T T(\mathrm{Suz}) \leq \mathbb{Z} / 2 \oplus \mathbb{Z} / 4$ since $X(N) \cong \mathbb{Z} / 2 \oplus \mathbb{Z} / 4$. Moreover, $G:=$ Suz has a maximal subgroup $H \cong J_{2}: 2$ containg the normaliser $N \cong 5^{2}:\left(4 \times \mathfrak{S}_{3}\right)$. Similar computations as above show that $H$ has two trivial source endotrivial modules of order 4, both affording the character $\chi_{18}+\chi_{19}$, with $\chi_{18}, \chi_{19} \in \operatorname{Irr}(H)$. The group $H$ also has a trivial source module of order 2 affording the character $\chi_{18}+\chi_{23}$ of degree 666 , hence not endotrivial. It follows that $\mathbb{Z} / 2 \leq T T(\mathrm{Suz}) \leq \mathbb{Z} / 4$. Finally a MAGMA computation shows that $T T(\mathrm{Suz}) \cong \mathbb{Z} / 2$.

6.14. The group $G=\mathrm{O}^{\prime} \mathrm{N}$ and its three-fold cover in characteristic 7 . In this case $P=7_{+}^{1+2}$ and $N \cong 7_{+}^{1+2}:\left(D_{8} \times 3\right)$ so that $X(N) \cong \mathbb{Z} / 2 \oplus \mathbb{Z} / 6$. The principal block is the unique block with full defect. Now $N$ is contained in a maximal subgroup $H \cong \mathrm{L}_{3}(7): 2$. First, let $1_{a} \in X(H) \cong \mathbb{Z} / 2$ be the non-trivial linear character of $H$. Then $e_{0} \cdot \operatorname{Ind}_{H}^{G}\left(1_{a}\right)=\chi_{10}$, which does not take value 1 on 7 -elements. Hence $\Gamma_{G}\left(1_{a}\right)$ is not endotrivial by Theorem 2.11. Since $T T(G)$ identifies with a subgroup of $T T(H)$ via restriction, it remains to show that $T T(H)=X(H)$. For this we let $1_{i} \in X(N)$, $1 \leq i \leq 12$ denote the non-trivial linear characters of $N$. The character $1_{6}$ has order 3 and $\operatorname{Ind}_{N}^{H}\left(1_{6}\right)=\chi_{7}+\chi_{9}$. The characters $1_{2}, 1_{4}$ have order 2 , the $k H$-Green correspondent of $1_{4}$ is $1_{a}$ and $\operatorname{Ind}_{N}^{H}\left(1_{2}\right)=\chi_{6}+\chi_{19}$. Therefore the characters of $\Gamma_{H}\left(1_{6}\right)$ and $\Gamma_{H}\left(1_{2}\right)$ cannot take value 1 on non-trivial 7 -elements, so that $\Gamma_{H}\left(1_{6}\right)$ and $\Gamma_{H}\left(1_{2}\right)$ are not endotrivial by Theorem 2.11. It follows that $T T(H)=X(H) \cong \mathbb{Z} / 2$ and $T T(G) \cong\{[k]\}$. Similarly to the situation for $G$, the three-fold cover 3.G $=3$. O'N has a maximal subgroup of shape $3 \times H$ containing $\widetilde{N}$. We have $X(\widetilde{N}) \cong \mathbb{Z} / 3 \oplus \mathbb{Z} / 6$ and $X(3 \times H) \cong \mathbb{Z} / 6$. Again since Green and Brauer correspondences commute, we only need to consider faithful modules. Let $1_{c}, 1_{d} \in X(3 \times H)$ be the two modules of order 6 . Inducing $1_{c}, 1_{d}$ to $3 . G$ in GAP, we get that the characters afforded by $\Gamma_{G}\left(1_{c}\right)$ and $\Gamma_{G}\left(1_{d}\right)$ are $\chi_{59}, \chi_{60} \in \operatorname{Irr}(3 . G)$. But both these characters take the value 15 on conjugacy class $7 a$, so that $\Gamma_{G}\left(1_{c}\right), \Gamma_{G}\left(1_{d}\right)$ cannot be endotrivial by Theorem 2.11. It follows that $T T(3 . G)$ has no faithful element of order 3 and so $T T(3 . G) \cong\{[k]\}$.

6.15. The group $G=\mathrm{Co}_{3}$ in characteristic 3. In this case $|P|=3^{7}$. We choose maximal subgroups $H_{1} \cong 3^{5}:\left(2 \times \mathrm{M}_{11}\right)$ and $H_{2} \cong 3^{1+4}:\left(4 . \mathfrak{S}_{6}\right)$ of $G$ both containing $N$ so that $T T(G) \leq T T\left(H_{1}\right)$ and $T T(G) \leq T T\left(H_{2}\right)$ via restriction. Since $\left|H_{1}: N\right|=55$ and $\left|H_{2}: N\right|=10$, trivial source endotrivial $k H_{1^{-}}, k H_{2}$-modules must be 1-dimensional by Lemma $2.2(7)$, that is $T T\left(H_{1}\right) \cong X\left(H_{1}\right) \cong \mathbb{Z} / 2$ and $T T\left(H_{2}\right) \cong X\left(H_{2}\right) \cong(\mathbb{Z} / 2)^{2}$. Then inducing the linear characters of $N$ in GAP, we find that there is a module $1_{a} \in X(N)$ such that $\Gamma_{H_{1}}\left(1_{a}\right) \in X\left(H_{1}\right)$ has order 2 , but $\Gamma_{H_{2}}\left(1_{a}\right)$ has dimension 10 and thus cannot be endotrivial. Whence $\Gamma_{G}\left(1_{a}\right)$ is not endotrivial either and $T T(G)=\{[k]\}$.

6.16. The group $G=\mathrm{Co}_{2}$ in characteristic 3 . In this case $|P|=3^{6}$. We choose maximal subgroups $H_{1} \cong 3^{5}:\left(2 \times \mathrm{M}_{11}\right)$ and $H_{2} \cong 3^{1+4}:\left(4 . \mathfrak{S}_{6}\right)$ of $G$ both containing $N$ 
so that $T T(G) \leq T T\left(H_{1}\right)$ and $T T(G) \leq T T\left(H_{2}\right)$ via restriction. Since $\left|H_{2}: N\right|=40$, trivial source endotrivial $k H_{2}$-modules must be 1-dimensional by Lemma 2.2(7) so that $T T\left(H_{2}\right) \cong X\left(H_{2}\right) \cong \mathbb{Z} / 2$. Let $1_{a} \in X(N)$ be the module such that $\Gamma_{H_{2}} \in X\left(H_{2}\right)$ is 1-dimensional of order 2 . Then

$$
\operatorname{Ind}_{N}^{H_{1}}\left(1_{a}\right)=\chi_{11}+\chi_{30}+\chi_{31}+\chi_{37}
$$

where $\chi_{11}, \chi_{30}, \chi_{31}, \chi_{37} \in \operatorname{Irr}\left(H_{1}\right)$ all take values greater than 1 on class $3 b$. Thus, by Theorem 2.11, $\Gamma_{H_{1}}\left(1_{a}\right)$ cannot be endotrivial, and neither can $\Gamma_{G}\left(1_{a}\right)$. Hence $T T(G)=$ $\{[k]\}$.

\subsection{The group $G=\mathrm{Fi}_{22}$ and its covers.}

Characteristic 3. In this case $P$ has order $3^{9}$, exponent 9 and is an extension of an elementary abelian 3-group of order $3^{5}$ with a Sylow 3 -subgroup of $\mathrm{U}_{4}(2)$, which is itself a wreath product $3 \prec 3$. Then $N$ is contained in a subgroup $H \cong \mathrm{O}_{7}(3)$ of $G$. By [CMN06, Theorem 6.2], we have $T T(H)=\{[k]\}$, and therefore $T T(G)=\{[k]\}$.

Characteristic 5. In this case $P \cong 5^{2}, N$ has order $2^{5} \cdot 3 \cdot 5^{2}$ and $X(N) \cong \mathbb{Z} / 4$. By [LMS13, Thm. 7.1], the character $\chi_{4} \in \operatorname{Irr}\left(\mathrm{Fi}_{22}\right)$ reduces modulo 5 to a self-dual endotrivial $k \mathrm{Fi}_{22}$-module $S_{1001}$ of dimension 1001, and by [LM14, Sec.2] we have $T T(G)=$ $\left\langle\left[S_{1001}\right]\right\rangle \cong \mathbb{Z} / 2$. Furthermore [LM14, Sec.2] also shows that $T T(2 . G) \cong \mathbb{Z} / 2 \oplus \mathbb{Z} / 2$, while $X(\widetilde{N}) \cong \mathbb{Z} / 2 \oplus \mathbb{Z} / 4$, and $T T(3 . G) \cong \mathbb{Z} / 6$, where by [LMS13, Thm. 7.1], the characters $\chi_{115}, \overline{\chi_{115}} \in \operatorname{Irr}\left(3 . \mathrm{Fi}_{22}\right)$ reduce modulo 5 to $k[3 . G]$-modules $S_{351}$ and $\left(S_{351}\right)^{*}$, both faithful simple trivial source endotrivial of dimension 351 . It follows that $T T(6 . G) \cong \mathbb{Z} / 6 \oplus \mathbb{Z} / 2$.

6.18. The group $G=\mathrm{HN}$.

Characteristic 3. In this case $|P|=3^{6}$ and we can choose maximal subgroups $H_{1} \cong$ $3^{4}: 2 .\left(\mathfrak{A}_{4} \times \mathfrak{A}_{4}\right) .4$ and $H_{2} \cong 3^{1+4}:\left(4 . \mathfrak{A}_{5}\right)$ of $G$, both containing $N$ so that $T T(G) \leq$ $T T\left(H_{1}\right)$ and $T T(G) \leq T T\left(H_{2}\right)$ via restriction. Since $\left|H_{1}: N\right|=16$ and $\left|H_{2}: N\right|=10$, trivial source endotrivial $k H_{1}$ - and $k H_{2}$-modules must be 1-dimensional by Lemma $2.2(7)$, that is $T T(G) \leq T T\left(H_{1}\right) \cong X\left(H_{1}\right) \cong \mathbb{Z} / 4$ and $T T(G) \leq T T\left(H_{2}\right) \cong X\left(H_{2}\right) \cong \mathbb{Z} / 2$. But if $1_{a} \in X(N)$ is of order 2 such that $\Gamma_{H_{2}}\left(1_{a}\right)$ is 1-dimensional, then $\Gamma_{H_{1}}\left(1_{a}\right)$ affords the irreducible character $\chi_{19} \in \operatorname{Irr}\left(H_{1}\right)$ of degree 16 , hence is not endotrivial and neither is $\Gamma_{G}\left(1_{a}\right)$. Whence $T T(G)=\{[k]\}$.

Characteristic 5. In this case $|P|=5^{6}$. Similarly as above, we choose maximal subgroups $H_{1} \cong 5^{1+4}: 2^{1+4} .5 .4$ and $H_{2} \cong 5^{2} \cdot 5 \cdot 5^{2} \cdot\left(4 . \mathfrak{A}_{5}\right)$ of $G$, both containing $N$. Since $\left|H_{1}: N\right|=16$ and $\left|H_{2}: N\right|=6$, we get $T T\left(H_{1}\right) \cong X\left(H_{1}\right) \cong \mathbb{Z} / 4$ and $T T\left(H_{2}\right) \cong X\left(H_{2}\right) \cong$ $\mathbb{Z} / 2$. Now if $1_{a} \in X(N)$ denotes the 1 -dimensional module such that $\Gamma_{H_{1}}\left(1_{a}\right) \in X\left(H_{1}\right)$ has order 2 , then inducing $1_{a}$ to $H_{2}$ at the level of characters yields $\operatorname{Ind}_{N}^{H_{2}}\left(1_{a}\right)=\chi_{7}+\chi_{8}$ where both $\chi_{7}, \chi_{8} \in \operatorname{Irr}\left(H_{2}\right)$ have degree 3 . Therefore $\Gamma_{H_{2}}\left(1_{a}\right)=\operatorname{Ind}_{N}^{H_{2}}\left(1_{a}\right)$ with dimension 6 and is not endotrivial. Whence $T T(G)=\{[k]\}$.

6.19. The group $G=\mathrm{Ly}$.

Characteristic 3. In this case $|P|=3^{7}$. We choose maximal subgroups $H_{1} \cong 3^{5}:(2 \times$ $\left.\mathrm{M}_{11}\right)$ and $H_{2} \cong 3^{2+4}: 2 . \mathfrak{A}_{5} . D_{8}$ both containing $N$. Since $\left|H_{1}: N\right|=55$ and $\left|H_{2}: N\right|=10$, we get $T T(G) \leq T T\left(H_{1}\right) \cong X\left(H_{1}\right) \cong \mathbb{Z} / 4$ and $T T(G) \leq T T\left(H_{2}\right) \cong X\left(H_{2}\right) \cong \mathbb{Z} / 2$. Thus an argument similar to that used for the simple group $H N$ applies and it follows that $T T(G)=\{[k]\}$. 
Characteristic 5. In this case $G$ contains a maximal subgroup $H \cong G_{2}(5)$, so that $T T(G)$ identifies with a subgroup of $T T(H)$ via restriction. Since $T T(H)=\{[k]\}$ by [CMN06, Thm. A], we have $T T(G)=\{[k]\}$.

6.20. The group $G=\mathrm{Fi}_{23}$ in characteristic 5 . In this case $P$ is elementary abelian of rank 2 and $X(N) \cong \mathbb{Z} / 2 \oplus \mathbb{Z} / 4$. By [LMS13, Thm. 7.1] the character $\chi_{9} \in \operatorname{Irr}\left(\mathrm{Fi}_{23}\right)$ reduces modulo 5 to a self-dual endotrivial $k \mathrm{Fi}_{23}$-module $S_{111826}$ of dimension 111826 . Therefore $\mathbb{Z} / 2 \leq T T(G) \leq \mathbb{Z} / 2 \oplus \mathbb{Z} / 4$. Now a maximal subgroup of $G$ is $H=2$.Fi in $_{22}$ and $H$ contains $N$, so that $T T(G) \leq T T\left(2 . \mathrm{Fi}_{22}\right) \cong(\mathbb{Z} / 2)^{2}$ via restriction. Moreover, if $S_{1001}$ denotes the self-dual simple endotrivial module of Subsection 6.17 of dimension 1001, we compute that $\Gamma_{G}\left(S_{1001}\right)$ affords $\chi_{5} \in \operatorname{Irr}(G)$ with degree $25806 \not \equiv 1(\bmod |P|)$. It follows that $T T(G) \cong \mathbb{Z} / 2$.

\subsection{The group $G=\mathrm{Co}_{1}$.}

Characteristic 3 . We choose maximal subgroups $H_{1} \cong 3^{1+4} \cdot 2$. $\mathrm{U}_{4}(2) .2$ and $H_{2} \cong 3^{6}$ : $2 . \mathrm{M}_{12}$ both containing $N$ and of index smaller than $|P|=3^{9}$. Then an argument similar to that used for the simple group $H N$ applies and it follows that $T T(G)=\{[k]\}$.

Characteristic 5. In this case $|P|=5^{4}$ and $X(N) \cong \mathbb{Z} / 4 \oplus \mathbb{Z} / 4$. Moreover the group $G$ has a maximal subgroup $H \cong 5^{1+2}: \mathrm{GL}_{2}(5)$ containing $N$ and such that $|H: N|=6$. Thus by Lemma $2.2(7), T T(G) \leq T T(H) \cong X(H) \cong \mathbb{Z} / 4$.

\subsection{The group $G=\mathrm{J}_{4}$.}

Characteristic 3. In this case $P=3_{+}^{1+2}, N=\left(2 \times 3_{+}^{1+2}: 8\right): 2$ and $X(N) \cong \mathbb{Z} / 2 \oplus \mathbb{Z} / 4$. We show that $T T(G)$ has no element of order 2 , hence is trivial. The group $G$ has a maximal subgroup $H \cong 2_{+}^{1+12} \cdot 3 \cdot M_{22}: 2$ containing $N$ and such that $X(H) \cong \mathbb{Z} / 2$. Let $1_{1}, 1_{2}, 1_{3}$ be the three linear characters of $N$ of order 2 . Then $\Gamma_{H}\left(1_{2}\right)=1_{a}$ the non-trivial linear character of $H$. The irreducible constituents of $\operatorname{Ind}_{N}^{H}\left(1_{1}\right)$ and $\operatorname{Ind}_{N}^{H}\left(1_{3}\right)$ all take a value greater than 1 on class $3 a$, so that by Theorem $2.11 \Gamma_{H}\left(1_{1}\right)$ and $\Gamma_{H}\left(1_{3}\right)$ are not endotrivial. Finally $e_{0} \cdot \operatorname{Ind}_{H}^{G}\left(1_{a}\right)=\chi_{40}+\chi_{52}$, where both $\chi_{40}, \chi_{52} \in \operatorname{Irr}(G)$ take value greater than 1 on conjugacy class $3 a$. Hence $\Gamma_{G}\left(1_{2}\right)$ is not endotrivial either. The claim follows.

Characteristic 11. The group $\mathrm{J}_{4}$ possesses a T.I. Sylow 11 -subgroup $P=11_{+}^{1+2}$ by Theorem 3.3, and we know that $N \cong 11_{+}^{1+2}:\left(5 \times\left(2 . \mathfrak{S}_{4}\right)\right)\left(\mathrm{cf}\left[\mathrm{CCN}^{+} 85\right]\right)$. Therefore $X(N) \cong \mathbb{Z} / 10$ and it follows from Lemma $2.8(4),(5)$ that $T T(G) \cong X(N) \cong \mathbb{Z} / 10$.

6.23. The group $G=\mathrm{B}$ in characteristic 5. The group B has a Sylow 5-subgroup of order $5^{6}$, whose normaliser $N$ is contained in a maximal subgroup $H \cong \mathrm{HN}: 2$. It follows directly from Subsection 6.18 that $T T(H) \cong \mathbb{Z} / 2$, hence $T T(G) \leq \mathbb{Z} / 2$. (We note that in this case $X(N) \cong \mathbb{Z} / 4 \oplus \mathbb{Z} / 4$.)

\section{Endotrivial MODUlES FOR SPORADIC GROUPS - SUMMARY OF RESULTS}

Table 3 below summarises the isomorphism types of the groups $T(G)$ of endotrivial $k G$-modules that we could determine in the case when the Sylow $p$-subgroups have $p$ rank at least 2. An empty entry means that the Sylow $p$-subgroups for the corresponding prime are either cyclic or trivial. A question mark indicates that only a partial result for the structure of $T T(G)$ has been obtained. In these cases we summarise the structure of the group $X(N)$ in Table 4, which we obtain using [GL83, GLS98, Bre12, BCP97]. The 
motivation for such computation is Lemma 2.8(2), asserting that $T T(G) \leq X(N)$. A question mark bounded by a group, e.g. $(? \leq \mathbb{Z} / 2)$ for the group B in characteristic 5 , indicates that we have found a sharper bound for $T T(G)$ than the one given by $X(N)$.

Finally, we assume that $G$ is quasi-simple with $G / Z(G)$ sporadic simple and a Sylow $p$ subgroup $P$ of $G$ is cyclic. Then $P \cong C_{p}$ and $p \neq 2\left(\right.$ see $\left.\left[\mathrm{CCN}^{+} 85\right]\right)$. We use Theorem 2.9 with, in our case, $H=N_{G}(P)$ and $e=\left|N_{G}(P): C_{G}(P)\right|$ is the inertial index of the principal block of $G$. The results come down to a routine computation using the structure of $H$ which can easily be computed using [GLS98, GAP13], and from which we get $X(H)$ and hence $T(G)$. We present them in Table 5 .

To compute $T(G)$ in this case, we recall that $T(G) \cong T(H)$, and the short exact sequence

$$
0 \longrightarrow X(H) \longrightarrow T(H) \stackrel{\operatorname{Res}_{P}^{H}}{\longrightarrow} T(P) \longrightarrow 0
$$

splits if and only if the class $\left[\Omega^{2}\left(k_{H}\right)\right]$ is a square in $X(H)$. In particular the sequence splits if $e$ is odd in which case $T(G) \cong X(H) \oplus \mathbb{Z} / 2$. Moreover $T(G)$ always contains a subgroup $\langle\Omega\rangle \cong \mathbb{Z} / 2 e$. If $|X(H)|=e$, then the principal block is the unique block containing endotrivial modules so that $T(G)=\langle\Omega\rangle \cong \mathbb{Z} / 2 e$. If $e$ is even, we use the distribution of endotrivial modules into blocks as described in [LMS13, Table 7]. Moreover, if m.G is a covering group of $G$ and $p \nmid m$, then $T(G)$ identifies with a subgroup of $T(m . G)$ via inflation. This is enough to conclude in all cases.

\section{REFERENCES}

[Bal13] P. Balmer. Modular representations of finite groups with trivial restriction to Sylow subgroups. J. Eur. Math. Soc. (JEMS), 15(6):2061-2079, 2013.

[BCP97] W. Bosma, J. Cannon, and C. Playoust. The Magma algebra system. I. The user language. J. Symbolic Comput., 24(3-4):235-265, 1997. Computational algebra and number theory (London, 1993).

[Ben81] D.J. Benson. The simple group $J_{4}$. PhD thesis, University of Cambridge, 1981.

[Bre12] T. Breuer. The GAP Character Table Library, Version 1.2.1. http://www.math.rwthaachen.de/ ${ }^{\sim}$ Thomas.Breuer/ctbllib, May 2012. GAP package.

[BS59] R. Brauer and M. Suzuki. On finite groups of even order whose 2-Sylow group is a quaternion group. Proc. Nat. Acad. Sci. U.S.A., 45:1757-1759, 1959.

[Car07] J. F. Carlson. Maximal elementary abelian subgroups of rank 2. J. Group Theory, 10(1):5-13, 2007.

[Car12] J. F. Carlson. Endotrivial modules. In Recent developments in Lie algebras, groups and representation theory, volume 86 of Proc. Sympos. Pure Math., pages 99-111. Amer. Math. Soc., Providence, RI, 2012.

$\left[\mathrm{CCN}^{+} 85\right]$ J. H. Conway, R. T. Curtis, S. P. Norton, R. A. Parker, and R. A. Wilson. Atlas of finite groups. Oxford University Press, Eynsham, 1985. Maximal subgroups and ordinary characters for simple groups, With computational assistance from J. G. Thackray.

[CHM10] J. F. Carlson, D. J. Hemmer, and N. Mazza. The group of endotrivial modules for the symmetric and alternating groups. Proc. Edinb. Math. Soc. (2), 53(1):83-95, 2010.

[CMN06] J. F. Carlson, N. Mazza, and D. K. Nakano. Endotrivial modules for finite groups of Lie type. J. Reine Angew. Math., 595:93-119, 2006.

[CMN09] J. F. Carlson, N. Mazza, and D. K. Nakano. Endotrivial modules for the symmetric and alternating groups. Proc. Edinb. Math. Soc. (2), 52(1):45-66, 2009. 
TABLE 3. $T(G)$ for covering groups of sporadic groups with $p^{2}$ dividing $|G|$

\begin{tabular}{|c|c|c|c|c|c|c|}
\hline Group $G$ & $p=2$ & $p=3$ & $p=5$ & $p=7$ & $p=11$ & $p=13$ \\
\hline 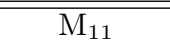 & 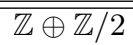 & $\overline{\bar{Z} \oplus(\mathbb{Z} / 2)^{2}}$ & & & & \\
\hline \multirow{2}{*}{$\begin{array}{c}\mathrm{M}_{12} \\
2 . \mathrm{M}_{12} \\
\end{array}$} & $\mathbb{Z}$ & $\mathbb{Z}^{3}$ & & & & \\
\hline & $\mathbb{Z}$ & $\cong T\left(M_{12}\right)$ & & & & \\
\hline$\overline{J_{1}}$ & $\overline{\mathbb{Z}}$ & & & & & \\
\hline \multirow{6}{*}{$\begin{array}{c}\mathrm{M}_{22} \\
2 . \mathrm{M}_{22} \\
3 . \mathrm{M}_{22} \\
4 . \mathrm{M}_{22} \\
6 . \mathrm{M}_{22} \\
12 . \mathrm{M}_{22}\end{array}$} & $\mathbb{Z}$ & $\bar{Z} \oplus(\mathbb{Z} / 2)^{2}$ & & & & \\
\hline & $\mathbb{Z}$ & $\mathbb{Z} \oplus \mathbb{Z} / 2 \oplus \mathbb{Z} / 4$ & & & & \\
\hline & $\mathbb{Z}$ & $\mathbb{Z}$ & & & & \\
\hline & $\mathbb{Z}$ & $\cong T\left(2 . \mathrm{M}_{22}\right)$ & & & & \\
\hline & $\mathbb{Z}$ & $\mathbb{Z}$ & & & & \\
\hline & $\mathbb{Z}$ & $\mathbb{Z}$ & & & & \\
\hline \multirow{2}{*}{$\begin{array}{c}\mathrm{J}_{2} \\
2 . \mathrm{J}_{2} \\
\end{array}$} & $\overline{\mathbb{Z}}$ & $\overline{\mathbb{Z} \oplus \mathbb{Z} / 2}$ & $\mathbb{Z} \oplus \mathbb{Z} / 2$ & & & \\
\hline & $\mathbb{Z}$ & $\cong T\left(\mathrm{~J}_{2}\right)$ & $\cong T\left(\mathrm{~J}_{2}\right)$ & & & \\
\hline 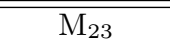 & $\overline{\mathbb{Z}}$ & $\overline{\mathbb{Z} \oplus \mathbb{Z} / 2}$ & & & & \\
\hline${ }^{2} \mathrm{~F}_{4}(2)^{\prime}$ & $\mathbb{Z}$ & $\mathbb{Z}^{2}$ & $\mathbb{Z} \oplus \mathbb{Z} / 6$ & & & \\
\hline \multirow{2}{*}{$\begin{array}{c}\mathrm{HS} \\
2 . \mathrm{HS} \\
\end{array}$} & $\overline{\bar{Z}}$ & $\overline{\bar{Z} \oplus(\mathbb{Z} / 2)^{2}}$ & $\overline{\mathbb{Z}^{2} \oplus \mathbb{Z} / 4}$ & & & \\
\hline & $\mathbb{Z}$ & $\cong T(\mathrm{HS})$ & $\cong T(\mathrm{HS})$ & & & \\
\hline \multirow{2}{*}{$\begin{array}{c}\mathrm{J}_{3} \\
3 . \mathrm{J}_{3} \\
\end{array}$} & $\mathbb{Z}$ & $\mathbb{Z} \oplus(? \leq \mathbb{Z} / 2)$ & & & & \\
\hline & $\mathbb{Z} \oplus \mathbb{Z} / 3$ & $\mathbb{Z}$ & & & & \\
\hline $\mathrm{M}_{24}$ & $\mathbb{Z}$ & $\mathbb{Z}^{2}$ & & & & \\
\hline \multirow{2}{*}{$\begin{array}{c}\mathrm{McL} \\
3 . \mathrm{McL} \\
\end{array}$} & $\overline{\mathbb{Z}}$ & $\overline{\mathbb{Z}}$ & $\mathbb{Z} \oplus \mathbb{Z} / 8$ & & & \\
\hline & $\mathbb{Z}$ & $\mathbb{Z}$ & $\mathbb{Z} \oplus \mathbb{Z} / 24$ & & & \\
\hline $\mathrm{He}$ & $\overline{\mathbb{Z}}$ & $\overline{\mathbb{Z}^{2}}$ & 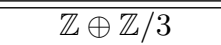 & $\mathbb{Z}^{3} \oplus ?$ & & \\
\hline \multirow{2}{*}{$\begin{array}{c}\mathrm{Ru} \\
2 . \mathrm{Ru} \\
\end{array}$} & $\mathbb{Z}$ & $\overline{\mathbb{Z} \oplus \mathbb{Z} / 2}$ & $\mathbb{Z}^{2} \oplus ?$ & & & \\
\hline & $\mathbb{Z}$ & $\mathbb{Z} \oplus \mathbb{Z} / 4$ & $\cong T(\mathrm{Ru})$ & & & \\
\hline \multirow{4}{*}{$\begin{array}{c}\text { Suz } \\
\text { 2.Suz } \\
\text { 3.Suz } \\
\text { 6.Suz }\end{array}$} & $\mathbb{Z}$ & $\mathbb{Z}$ & $\mathbb{Z} \oplus \mathbb{Z} / 2$ & & & \\
\hline & $\mathbb{Z}$ & $\cong T(\mathrm{Suz})$ & $\cong T(\mathrm{Suz})$ & & & \\
\hline & $\mathbb{Z}$ & $\mathbb{Z}$ & $\cong T(\mathrm{Suz})$ & & & \\
\hline & $\mathbb{Z}$ & $\mathbb{Z}$ & $\cong T(\mathrm{Suz})$ & & & \\
\hline \multirow{2}{*}{$\begin{array}{c}\mathrm{O}^{\prime} \mathrm{N} \\
3 . \mathrm{O}^{\prime} \mathrm{N} \\
\end{array}$} & $\mathbb{Z}$ & $\overline{\mathbb{Z} \oplus(? \leq \mathbb{Z} / 2)}$ & & $\mathbb{Z}^{3}$ & & \\
\hline & $\mathbb{Z}$ & $\mathbb{Z}$ & & $\mathbb{Z}^{3}$ & & \\
\hline $\mathrm{Co}_{3}$ & $\mathbb{Z}$ & $\mathbb{Z}$ & $\mathbb{Z} \oplus ?$ & & & \\
\hline $\mathrm{Co}_{2}$ & $\mathbb{Z}$ & $\mathbb{Z}$ & $\mathbb{Z} \oplus ?$ & & & \\
\hline \multirow{4}{*}{$\begin{array}{c}\mathrm{Fi}_{22} \\
2 . \mathrm{Fi}_{22} \\
3 . \mathrm{Fi}_{22} \\
\text { 6. } \mathrm{Fi}_{22}\end{array}$} & $\overline{\mathbb{Z}}$ & $\begin{array}{c}\mathbb{Z} \\
\end{array}$ & 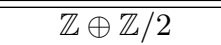 & & & \\
\hline & $\mathbb{Z}$ & $\cong T\left(\mathrm{Fi}_{22}\right)$ & $\mathbb{Z} \oplus(\mathbb{Z} / 2)^{2}$ & & & \\
\hline & $\mathbb{Z}$ & $\mathbb{Z}$ & $\mathbb{Z} \oplus \mathbb{Z} / 6$ & & & \\
\hline & $\mathbb{Z}$ & $\mathbb{Z}$ & $\mathbb{Z} \oplus \mathbb{Z} / 6 \oplus \mathbb{Z} / 2$ & & & \\
\hline $\mathrm{F}_{5}=\mathrm{HN}$ & $\mathbb{Z}$ & $\mathbb{Z}$ & $\mathbb{Z}$ & & & \\
\hline Ly & $\overline{\mathbb{Z}}$ & $\overline{\mathbb{Z}}$ & $\overline{\mathbb{Z}}$ & & & \\
\hline Th & $\mathbb{Z}$ & $\mathbb{Z} \oplus ?$ & $\mathbb{Z} \oplus ?$ & $\mathbb{Z} \oplus ?$ & & \\
\hline $\mathrm{Fi}_{23}$ & $\mathbb{Z}$ & $\mathbb{Z} \oplus ?$ & $\mathbb{Z} \oplus \mathbb{Z} / 2$ & & & \\
\hline \multirow{2}{*}{$\begin{array}{c}\mathrm{Co}_{1} \\
2 . \mathrm{Co}_{1} \\
\end{array}$} & $\overline{\mathbb{Z}}$ & $\overline{\mathbb{Z}}$ & $\overline{\mathbb{Z}^{2} \oplus(? \leq \mathbb{Z} / 4)}$ & $\mathbb{Z} \oplus ?$ & & \\
\hline & $\mathbb{Z}$ & $\cong T\left(\mathrm{Co}_{1}\right)$ & $\cong T\left(\mathrm{Co}_{1}\right)$ & $\cong T\left(\mathrm{Co}_{1}\right)$ & & \\
\hline $\mathrm{J}_{4}$ & $\mathbb{Z}$ & $\mathbb{Z}$ & & & $\mathbb{Z} \oplus \mathbb{Z} / 10$ & \\
\hline \multirow{2}{*}{$\begin{array}{c}\mathrm{Fi}_{24}^{\prime} \\
\text { 3. } \mathrm{Fi}_{24}^{\prime}\end{array}$} & $\mathbb{Z}$ & $\mathbb{Z} \oplus ?$ & $\mathbb{Z} \oplus ?$ & $\mathbb{Z}^{3} \oplus ?$ & & \\
\hline & $\mathbb{Z}$ & $\mathbb{Z}$ & $\cong T\left(\mathrm{Fi}_{24}^{\prime}\right)$ & $\cong T\left(\mathrm{Fi}_{24}^{\prime}\right)$ & & \\
\hline \multirow{3}{*}{$\begin{array}{c}\mathrm{F}_{2}=\mathrm{B} \\
2 . \mathrm{F}_{2}=2 . \mathrm{B} \\
\mathrm{F}_{1}=\mathrm{M}\end{array}$} & $\mathbb{Z}$ & 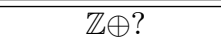 & $\overline{\mathbb{Z} \oplus(? \leq \mathbb{Z} / 2)}$ & 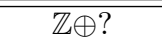 & & \\
\hline & $\mathbb{Z}$ & $\cong T(\mathrm{~B})$ & $\cong T(\mathrm{~B})$ & $\cong T(\mathrm{~B})$ & & \\
\hline & $\mathbb{Z}$ & $\mathbb{Z} \oplus ?$ & $\mathbb{Z} \oplus ?$ & $\mathbb{Z} \oplus ?$ & $\mathbb{Z} \oplus ?$ & $\mathbb{Z}^{2} \oplus$ \\
\hline
\end{tabular}


TABLE 4. The structure of $X(N)$ for the missing cases in Table 3

\begin{tabular}{|c|c|c||c|c|c||c|c|c|}
\hline$G$ & $p$ & $X(N)$ & $G$ & $p$ & $X(N)$ & $G$ & $p$ & $X(N)$ \\
\hline $\mathrm{He}$ & 7 & $\mathbb{Z} / 6$ & $\mathrm{Th}$ & 7 & $\mathbb{Z} / 6$ & $\mathrm{~B}$ & 7 & $\mathbb{Z} / 2 \oplus \mathbb{Z} / 6$ \\
$\mathrm{Ru}$ & 5 & $\mathbb{Z} / 2 \oplus \mathbb{Z} / 4$ & $\mathrm{Fi}_{23}$ & 3 & $(\mathbb{Z} / 2)^{3}$ & $\mathrm{M}$ & 3 & $(\mathbb{Z} / 2)^{3}$ \\
$\mathrm{O}^{\prime} \mathrm{N}$ & 3 & $\mathbb{Z} / 2$ & $\mathrm{Co}_{1}$ & 7 & $\mathbb{Z} / 3 \oplus \mathbb{Z} / 3$ & $\mathrm{M}$ & 5 & $\mathbb{Z} / 2 \oplus(\mathbb{Z} / 4)^{2}$ \\
$\mathrm{Co}_{3}$ & 5 & $\mathbb{Z} / 2 \oplus \mathbb{Z} / 4$ & $\mathrm{Fi}_{24}^{\prime}$ & 3 & $(\mathbb{Z} / 2)^{3}$ & $\mathrm{M}$ & 7 & $\mathbb{Z} / 2 \oplus \mathbb{Z} / 6$ \\
$\mathrm{Co}_{2}$ & 5 & $\mathbb{Z} / 4$ & $\mathrm{Fi}_{24}^{\prime}$ & 5 & $\mathbb{Z} / 4$ & $\mathrm{M}$ & 11 & $\mathbb{Z} / 5$ \\
$\mathrm{Th}$ & 3 & $(\mathbb{Z} / 2)^{2}$ & $\mathrm{Fi}_{24}^{\prime}$ & 7 & $\mathbb{Z} / 2 \oplus \mathbb{Z} / 6$ & $\mathrm{M}$ & 13 & $\mathbb{Z} / 12$ \\
$\mathrm{Th}$ & 5 & $\mathbb{Z} / 4$ & $\mathrm{~B}$ & 3 & $(\mathbb{Z} / 2)^{3}$ & & & \\
\hline
\end{tabular}

[CMT11] J. F. Carlson, N. Mazza, and J. Thévenaz. Endotrivial modules for $p$-solvable groups. Trans. Amer. Math. Soc., 363(9):4979-4996, 2011.

[CMT13] J. F. Carlson, N. Mazza, and J. Thévenaz. Endotrivial modules over groups with quaternion or semi-dihedral Sylow 2-subgroup. J. Eur. Math. Soc. (JEMS), 15(1):157-177, 2013.

[Dad78a] E. C. Dade. Endo-permutation modules over p-groups. I. Ann. of Math, 107:459-494, 1978.

[Dad78b] E. C. Dade. Endo-permutation modules over p-groups. II. Ann. of Math, 108:317-346, 1978.

[DK09] S. Danz and B. Külshammer. Vertices, sources and Green correspondents of the simple modules for the large mathieu groups. J. Algebra, 322(11):317-346, 2009.

[GAP13] The GAP Group. GAP - Groups, Algorithms, and Programming, Version 4.6.5, 2013.

[GL83] D. Gorenstein and R. Lyons. The local structure of finite groups of characteristic 2 type. Mem. Amer. Math. Soc., 42(276):vii+731, 1983.

[GLS98] D. Gorenstein, R. Lyons, and R. Solomon. The classification of the finite simple groups. Number 3. Part I. Chapter A, volume 40 of Mathematical Surveys and Monographs. American Mathematical Society, Providence, RI, 1998. Almost simple $K$-groups.

[GM10] G. Glauberman and N. Mazza. p-groups with maximal elementary abelian subgroups of rank 2. J. Algebra, 323(6):1729-1737, 2010.

[Kaw93] S. Kawata. On Auslander-Reiten components for certain group modules. Osaka J. Math., 30(2):137-157, 1993.

[KW99a] S. Koshitani and K. Waki. The Green correspondents of the Mathieu group $M_{12}$ in characteristic 3. Comm. Algebra, 27(1):37-66, 1999.

[KW99b] S. Koshitani and K. Waki. The Loewy structure of the projective modules of the Mathieu group $M_{12}$ and its automorphism group in characteristic 3. Comm. Algebra, 27(1):1-36, 1999.

[Lan83] P. Landrock. Finite Group Algebras and their Modules. In Finite Group Algebras and their Modules, volume 84 of London Mathematical Society Lecture Note Series, pages x+274. Cambridge University Press, Cambridge, 1983.

[LM78] P. Landrock and G. O. Michler. Block structure of the smallest Janko group. Math. Ann., 232(3):205-238, 1978.

[LM14] C. Lassueur and G. Malle. Simple endotrivial modules for quasi-simple groups II. Prepint, 2014.

[LMS13] C. Lassueur, G. Malle, and E. Schulte. Simple endotrivial modules for quasi-simple groups. To appear in J. Reine Angew. Math., pages 1-31, 2013. http://arxiv.org/pdf/1305.3466.pdf.

[Mac70] A. R. MacWilliams. On 2-groups with no normal abelian subgroups of rank 3, and their occurrence as Sylow 2-subgroups of finite simple groups. Trans. Amer. Math. Soc., 150:345408, 1970.

[Maz08] N. Mazza. Connected components of the category of elementary abelian p-subgroups. J. Algebra, 320(12):4242-4248, 2008. 
TABLE 5. $T(G)$ for covering groups of sporadic groups with cyclic Sylow $p$-subgroups

\begin{tabular}{|c|c|c|c|c|c|c|c|c|c|}
\hline$G$ & $p$ & $X(H)$ & $e$ & $T(G)$ & $G$ & $p$ & $X(H)$ & $e$ & $T(G)$ \\
\hline${ }^{2} F_{4}(2)^{\prime}$ & 13 & $\mathbb{Z} / 6$ & 6 & $\overline{\mathbb{Z} / 12}$ & $\mathrm{He}$ & 17 & $\mathbb{Z} / 8$ & 8 & $\mathbb{Z} / 16$ \\
\hline $\mathrm{M}_{11}$ & 5 & $\mathbb{Z} / 4$ & 4 & $\mathbb{Z} / 8$ & $\mathrm{Ru}$ & 7 & $\mathbb{Z} / 6$ & 6 & $\mathbb{Z} / 12$ \\
\hline $\mathrm{M}_{11}$ & 11 & $\mathbb{Z} / 5$ & 5 & $\mathbb{Z} / 10$ & 2.Ru & 7 & $\mathbb{Z} / 6$ & 6 & $\cong T(\mathrm{Ru})$ \\
\hline $\mathrm{M}_{12}$ & 5 & $\mathbb{Z} / 2 \oplus \mathbb{Z} / 4$ & 4 & $\mathbb{Z} / 2 \oplus \mathbb{Z} / 8$ & $\mathrm{Ru}$ & 13 & $\mathbb{Z} / 12$ & 12 & $\mathbb{Z} / 24$ \\
\hline 2. $\mathrm{M}_{12}$ & 5 & $\mathbb{Z} / 2 \oplus \mathbb{Z} / 4$ & 4 & $\cong T\left(\mathrm{M}_{12}\right)$ & 2. $\mathrm{Ru}$ & 13 & $\mathbb{Z} / 12$ & 12 & $\cong T(\mathrm{Ru})$ \\
\hline $\mathrm{M}_{12}$ & 11 & $\mathbb{Z} / 5$ & 5 & $\mathbb{Z} / 10$ & $\mathrm{Ru}$ & 29 & $\mathbb{Z} / 14$ & 14 & $\mathbb{Z} / 28$ \\
\hline 2. $\mathrm{M}_{12}$ & 11 & $\mathbb{Z} / 10$ & 5 & $\mathbb{Z} / 2 \oplus \mathbb{Z} / 10$ & 2. $\mathrm{Ru}$ & 29 & $\mathbb{Z} / 28$ & 14 & $\mathbb{Z} / 2 \oplus \mathbb{Z} / 28$ \\
\hline $\mathrm{J}_{1}$ & 3 & $\mathbb{Z} / 2 \oplus \mathbb{Z} / 2$ & 2 & $\mathbb{Z} / 2 \oplus \mathbb{Z} / 4$ & Suz & 7 & $\mathbb{Z} / 6$ & 6 & $\mathbb{Z} / 12$ \\
\hline $\mathrm{J}_{1}$ & 5 & $\mathbb{Z} / 2 \oplus \mathbb{Z} / 2$ & 2 & $\mathbb{Z} / 2 \oplus \mathbb{Z} / 4$ & 2.Suz & 7 & $\mathbb{Z} / 6$ & 6 & $\cong T(\mathrm{Suz})$ \\
\hline $\mathrm{J}_{1}$ & 7 & $\mathbb{Z} / 6$ & 6 & $\mathbb{Z} / 12$ & 3.Suz & 7 & $\mathbb{Z} / 3 \oplus \mathbb{Z} / 6$ & 6 & $\mathbb{Z} / 3 \oplus \mathbb{Z} / 12$ \\
\hline $\mathrm{J}_{1}$ & 11 & $\mathbb{Z} / 10$ & 10 & $\mathbb{Z} / 20$ & 6.Suz & 7 & $\mathbb{Z} / 3 \oplus \mathbb{Z} / 6$ & 6 & $\cong T(3 . \mathrm{Suz})$ \\
\hline $\mathrm{J}_{1}$ & 19 & $\mathbb{Z} / 6$ & 6 & $\mathbb{Z} / 12$ & Suz & 11 & $\mathbb{Z} / 10$ & 10 & $\mathbb{Z} / 20$ \\
\hline $\mathrm{M}_{22}$ & 5 & $\mathbb{Z} / 4$ & 4 & $\mathbb{Z} / 8$ & 2.Suz & 11 & $\mathbb{Z} / 20$ & 10 & $\mathbb{Z} / 2 \oplus \mathbb{Z} / 20$ \\
\hline 2. $\mathrm{M}_{22}$ & 5 & $\mathbb{Z} / 2 \oplus \mathbb{Z} / 4$ & 4 & $\mathbb{Z} / 2 \oplus \mathbb{Z} / 8$ & 3.Suz & 11 & $\mathbb{Z} / 30$ & 10 & $\mathbb{Z} / 60$ \\
\hline 3. $\mathrm{M}_{22}$ & 5 & $\mathbb{Z} / 12$ & 4 & $\mathbb{Z} / 24$ & 6.Suz & 11 & $\mathbb{Z} / 60$ & 10 & $\mathbb{Z} / 2 \oplus \mathbb{Z} / 60$ \\
\hline 4. $\mathrm{M}_{22}$ & 5 & $\mathbb{Z} / 2 \oplus \mathbb{Z} / 8$ & 4 & $\mathbb{Z} / 4 \oplus \mathbb{Z} / 8$ & Suz & 13 & $\mathbb{Z} / 6$ & 6 & $\mathbb{Z} / 12$ \\
\hline $6 . \mathrm{M}_{22}$ & 5 & $\mathbb{Z} / 2 \oplus \mathbb{Z} / 12$ & 4 & $\mathbb{Z} / 2 \oplus \mathbb{Z} / 24$ & 2.Suz & 13 & $\mathbb{Z} / 12$ & 6 & $\mathbb{Z} / 2 \oplus \mathbb{Z} / 12$ \\
\hline $12 . \mathrm{M}_{22}$ & 5 & $\mathbb{Z} / 2 \oplus \mathbb{Z} / 24$ & 4 & $\mathbb{Z} / 4 \oplus \mathbb{Z} / 24$ & 3.Suz & 13 & $\mathbb{Z} / 3 \oplus \mathbb{Z} / 6$ & 6 & $\mathbb{Z} / 3 \oplus \mathbb{Z} / 12$ \\
\hline $\mathrm{M}_{22}$ & 7 & $\mathbb{Z} / 3$ & 3 & $\mathbb{Z} / 6$ & 6.Suz & 13 & $\mathbb{Z} / 3 \oplus \mathbb{Z} / 12$ & 6 & $\mathbb{Z} / 6 \oplus \mathbb{Z} / 12$ \\
\hline 2. $\mathrm{M}_{22}$ & 7 & $\mathbb{Z} / 6$ & 3 & $\mathbb{Z} / 2 \oplus \mathbb{Z} / 6$ & $\mathrm{ON}$ & 5 & $\mathbb{Z} / 2 \oplus \mathbb{Z} / 4$ & 4 & $\mathbb{Z} / 2 \oplus \mathbb{Z} / 8$ \\
\hline $3 . \mathrm{M}_{22}$ & 7 & $\mathbb{Z} / 3 \oplus \mathbb{Z} / 3$ & 3 & $\mathbb{Z} / 3 \oplus \mathbb{Z} / 6$ & $3 . \mathrm{ON}$ & 5 & $\mathbb{Z} / 2 \oplus \mathbb{Z} / 4$ & 4 & $\cong T(\mathrm{ON})$ \\
\hline 4. $\mathrm{M}_{22}$ & 7 & $\mathbb{Z} / 12$ & 3 & $\mathbb{Z} / 2 \oplus \mathbb{Z} / 12$ & $\mathrm{ON}$ & 11 & $\mathbb{Z} / 10$ & 10 & $\mathbb{Z} / 20$ \\
\hline $6 \cdot \mathrm{M}_{22}$ & 7 & $\mathbb{Z} / 3 \oplus \mathbb{Z} / 6$ & 3 & $\mathbb{Z} / 6 \oplus \mathbb{Z} / 6$ & $3 . \mathrm{ON}$ & 11 & $\mathbb{Z} / 30$ & 10 & $\mathbb{Z} / 60$ \\
\hline $12 . \mathrm{M}_{22}$ & 7 & $\mathbb{Z} / 3 \oplus \mathbb{Z} / 12$ & 3 & $\mathbb{Z} / 6 \oplus \mathbb{Z} / 12$ & $\mathrm{ON}$ & 19 & $\mathbb{Z} / 6$ & 6 & $\mathbb{Z} / 12$ \\
\hline $\mathrm{M}_{22}$ & 11 & $\mathbb{Z} / 5$ & 5 & $\mathbb{Z} / 10$ & $3 . \mathrm{ON}$ & 19 & $\mathbb{Z} / 3 \oplus \mathbb{Z} / 6$ & 6 & $\mathbb{Z} / 3 \oplus \mathbb{Z} / 12$ \\
\hline $2 . \mathrm{M}_{22}$ & 11 & $\mathbb{Z} / 10$ & 5 & $\mathbb{Z} / 2 \oplus \mathbb{Z} / 10$ & $\mathrm{ON}$ & 31 & $\mathbb{Z} / 15$ & 15 & $\mathbb{Z} / 30$ \\
\hline 3. $\mathrm{M}_{22}$ & 11 & $\mathbb{Z} / 15$ & 5 & $\mathbb{Z} / 30$ & $3 . \mathrm{ON}$ & 31 & $\mathbb{Z} / 3 \oplus \mathbb{Z} / 15$ & 15 & $\mathbb{Z} / 3 \oplus \mathbb{Z} / 30$ \\
\hline 4. $\mathrm{M}_{22}$ & 11 & $\mathbb{Z} / 20$ & 5 & $\mathbb{Z} / 2 \oplus \mathbb{Z} / 20$ & $\mathrm{Co}_{3}$ & 7 & $\mathbb{Z} / 2 \oplus \mathbb{Z} / 6$ & 6 & $\mathbb{Z} / 2 \oplus \mathbb{Z} / 12$ \\
\hline $6 . \mathrm{M}_{22}$ & 11 & $\mathbb{Z} / 30$ & 5 & $\mathbb{Z} / 2 \oplus \mathbb{Z} / 30$ & $\mathrm{Co}_{3}$ & 11 & $\mathbb{Z} / 10$ & 5 & $\mathbb{Z} / 2 \oplus \mathbb{Z} / 10$ \\
\hline $12 . \mathrm{M}_{22}$ & 11 & $\mathbb{Z} / 60$ & 5 & $\mathbb{Z} / 2 \oplus \mathbb{Z} / 60$ & $\mathrm{Co}_{3}$ & 23 & $\mathbb{Z} / 11$ & 11 & $\mathbb{Z} / 22$ \\
\hline $\mathrm{J}_{2}$ & 7 & $\mathbb{Z} / 6$ & 6 & $\mathbb{Z} / 12$ & $\mathrm{Co}_{2}$ & 7 & $\mathbb{Z} / 2 \oplus \mathbb{Z} / 6$ & 6 & $\mathbb{Z} / 2 \oplus \mathbb{Z} / 12$ \\
\hline 2. $\mathrm{J}_{2}$ & 7 & $\mathbb{Z} / 12$ & 6 & $\mathbb{Z} / 2 \oplus \mathbb{Z} / 12$ & $\mathrm{Co}_{2}$ & 11 & $\mathbb{Z} / 10$ & 10 & $\mathbb{Z} / 20$ \\
\hline $\mathrm{M}_{23}$ & 5 & $\mathbb{Z} / 4$ & 4 & $\mathbb{Z} / 8$ & $\mathrm{Co}_{2}$ & 23 & $\mathbb{Z} / 11$ & 11 & $\mathbb{Z} / 22$ \\
\hline $\mathrm{M}_{23}$ & 7 & $\mathbb{Z} / 6$ & 3 & $\mathbb{Z} / 2 \oplus \mathbb{Z} / 6$ & $\mathrm{Fi}_{2} 2$ & 7 & $\mathbb{Z} / 2 \oplus \mathbb{Z} / 6$ & 6 & $\mathbb{Z} / 2 \oplus \mathbb{Z} / 12$ \\
\hline $\mathrm{M}_{23}$ & 11 & $\mathbb{Z} / 5$ & 5 & $\mathbb{Z} / 10$ & 2. $\mathrm{Fi}_{2} 2$ & 7 & $\mathbb{Z} / 2 \oplus \mathbb{Z} / 2 \oplus \mathbb{Z} / 6$ & 6 & $\mathbb{Z} / 2 \oplus \mathbb{Z} / 2 \oplus \mathbb{Z} / 12$ \\
\hline $\mathrm{M}_{23}$ & 23 & $\mathbb{Z} / 11$ & 11 & $\mathbb{Z} / 22$ & 3. $\mathrm{Fi}_{2} 2$ & 7 & $\mathbb{Z} / 6 \oplus \mathbb{Z} / 6$ & 6 & $\mathbb{Z} / 6 \oplus \mathbb{Z} / 12$ \\
\hline $\mathrm{HS}$ & 7 & $\mathbb{Z} / 6$ & 6 & $\mathbb{Z} / 12$ & 6. $\mathrm{Fi}_{2} 2$ & 7 & $\mathbb{Z} / 2 \oplus \mathbb{Z} / 6 \oplus \mathbb{Z} / 6$ & 6 & $\mathbb{Z} / 2 \oplus \mathbb{Z} / 6 \oplus \mathbb{Z} / 12$ \\
\hline 2.HS & 7 & $\mathbb{Z} / 12$ & 6 & $\mathbb{Z} / 2 \oplus \mathbb{Z} / 12$ & $\mathrm{Fi}_{2} 2$ & 11 & $\mathbb{Z} / 10$ & 5 & $\mathbb{Z} / 2 \oplus \mathbb{Z} / 10$ \\
\hline HS & 11 & $\mathbb{Z} / 5$ & 5 & $\mathbb{Z} / 10$ & 2. $\mathrm{Fi}_{2} 2$ & 11 & $\mathbb{Z} / 2 \oplus \mathbb{Z} / 10$ & 5 & $\mathbb{Z} / 2 \oplus \mathbb{Z} / 2 \oplus \mathbb{Z} / 10$ \\
\hline 2.HS & 11 & $\mathbb{Z} / 10$ & 5 & $\mathbb{Z} / 2 \oplus \mathbb{Z} / 10$ & 3. $\mathrm{Fi}_{2} 2$ & 11 & $\mathbb{Z} / 30$ & 5 & $\mathbb{Z} / 2 \oplus \mathbb{Z} / 30$ \\
\hline $\mathrm{J}_{3}$ & 5 & $\mathbb{Z} / 2 \oplus \mathbb{Z} / 2$ & 2 & $\mathbb{Z} / 2 \oplus \mathbb{Z} / 4$ & 6. $\mathrm{Fi}_{2} 2$ & 11 & $\mathbb{Z} / 2 \oplus \mathbb{Z} / 30$ & 5 & $\mathbb{Z} / 2 \oplus \mathbb{Z} / 2 \oplus \mathbb{Z} / 30$ \\
\hline 3. $\mathrm{J}_{3}$ & 5 & $\mathbb{Z} / 2 \oplus \mathbb{Z} / 6$ & 2 & $\mathbb{Z} / 2 \oplus \mathbb{Z} / 12$ & $\mathrm{Fi}_{2} 2$ & 13 & $\mathbb{Z} / 6$ & 6 & $\mathbb{Z} / 12$ \\
\hline $\mathrm{J}_{3}$ & 17 & $\mathbb{Z} / 8$ & 8 & $\mathbb{Z} / 16$ & 2. $\mathrm{Fi}_{2} 2$ & 13 & $\mathbb{Z} / 2 \oplus \mathbb{Z} / 6$ & 6 & $\mathbb{Z} / 2 \oplus \mathbb{Z} / 12$ \\
\hline 3. $\mathrm{J}_{3}$ & 17 & $\mathbb{Z} / 24$ & 8 & $\mathbb{Z} / 48$ & 3. $\mathrm{Fi}_{2} 2$ & 13 & $\mathbb{Z} / 3 \oplus \mathbb{Z} / 6$ & 6 & $\mathbb{Z} / 3 \oplus \mathbb{Z} / 12$ \\
\hline $\mathrm{J}_{3}$ & 19 & $\mathbb{Z} / 9$ & 9 & $\mathbb{Z} / 18$ & 6. $\mathrm{Fi}_{2} 2$ & 13 & $\mathbb{Z} / 6 \oplus \mathbb{Z} / 6$ & 6 & $\mathbb{Z} / 6 \oplus \mathbb{Z} / 12$ \\
\hline 3. $\mathrm{J}_{3}$ & 19 & $\mathbb{Z} / 3 \oplus \mathbb{Z} / 9$ & 9 & $\mathbb{Z} / 3 \oplus \mathbb{Z} / 18$ & $\mathrm{HN}$ & 7 & $\mathbb{Z} / 6$ & 6 & $\mathbb{Z} / 12$ \\
\hline $\mathrm{M}_{24}$ & 5 & $\mathbb{Z} / 4$ & 4 & $\mathbb{Z} / 8$ & $\mathrm{HN}$ & 11 & $\mathbb{Z} / 2 \oplus \mathbb{Z} / 10$ & 10 & $\mathbb{Z} / 2 \oplus \mathbb{Z} / 20$ \\
\hline $\mathrm{M}_{24}$ & 7 & $\mathbb{Z} / 6$ & 3 & $\mathbb{Z} / 2 \oplus \mathbb{Z} / 6$ & $\mathrm{HN}$ & 19 & $\mathbb{Z} / 9$ & 9 & $\mathbb{Z} / 18$ \\
\hline $\mathrm{M}_{24}$ & 11 & $\mathbb{Z} / 10$ & 10 & $\mathbb{Z} / 20$ & Ly & 7 & $\mathbb{Z} / 6$ & 6 & $\mathbb{Z} / 12$ \\
\hline $\mathrm{M}_{24}$ & 23 & $\mathbb{Z} / 11$ & 11 & $\mathbb{Z} / 22$ & Ly & 11 & $\mathbb{Z} / 10$ & 5 & $\mathbb{Z} / 2 \oplus \mathbb{Z} / 10$ \\
\hline $\mathrm{McL}$ & 7 & $\mathbb{Z} / 6$ & 3 & $\mathbb{Z} / 2 \oplus \mathbb{Z} / 6$ & Ly & 31 & $\mathbb{Z} / 6$ & 6 & $\mathbb{Z} / 12$ \\
\hline 3.McL & 7 & $\mathbb{Z} / 3 \oplus \mathbb{Z} / 6$ & 3 & $\mathbb{Z} / 6 \oplus \mathbb{Z} / 6$ & Ly & 37 & $\mathbb{Z} / 18$ & 18 & $\mathbb{Z} / 36$ \\
\hline $\mathrm{McL}$ & 11 & $\mathbb{Z} / 5$ & 5 & $\mathbb{Z} / 10$ & Ly & 67 & $\mathbb{Z} / 22$ & 22 & $\mathbb{Z} / 44$ \\
\hline 3.McL & 11 & $\mathbb{Z} / 15$ & 5 & $\mathbb{Z} / 30$ & $\mathrm{Th}$ & 13 & $\mathbb{Z} / 12$ & 12 & $\mathbb{Z} / 24$ \\
\hline
\end{tabular}




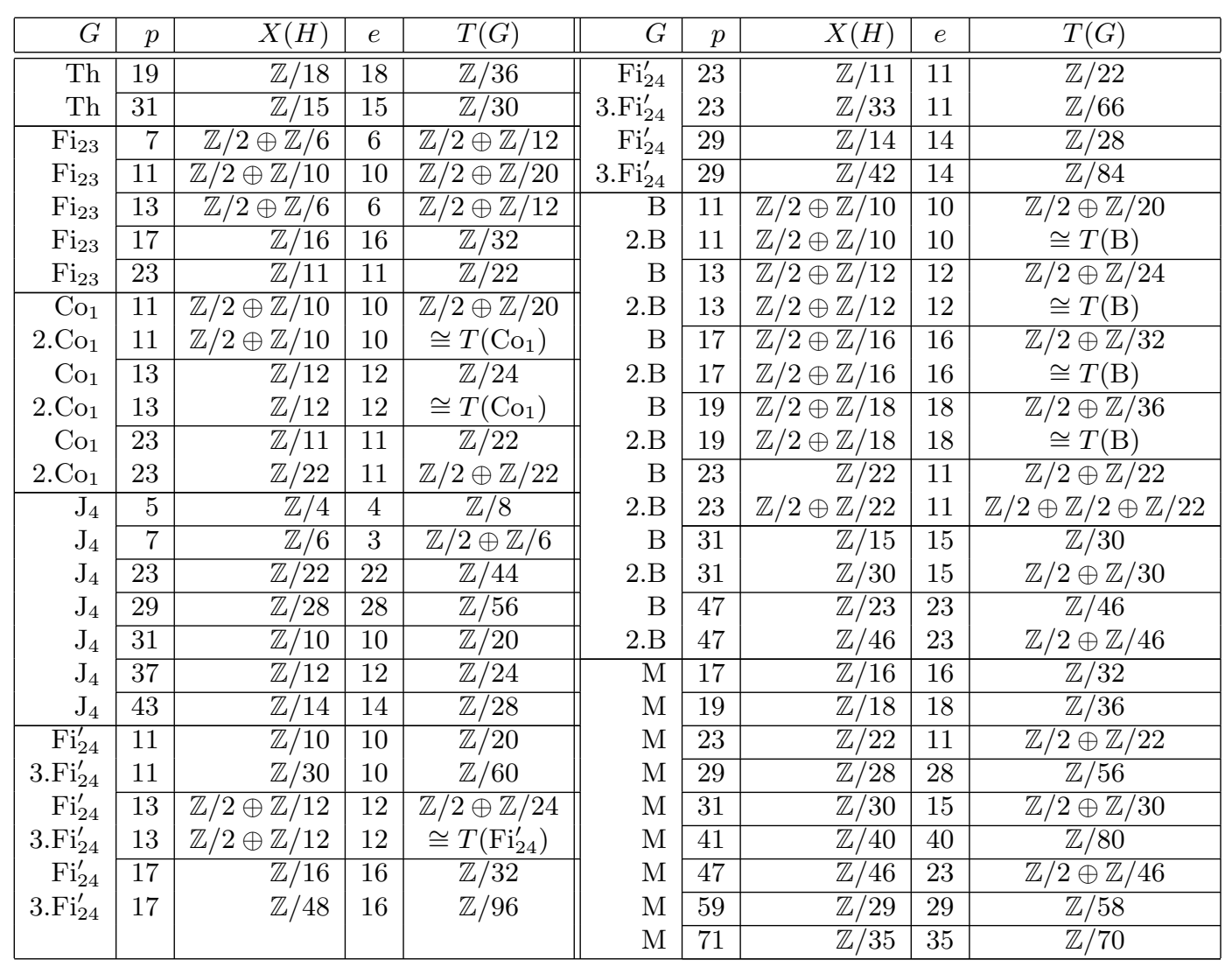

[Mic91] G. O. Michler. Contributions to modular representation theory of finite groups. In Representation theory of finite groups and finite-dimensional algebras (Bielefeld, 1991), volume 95 of Progr. Math., pages 99-140. Birkhäuser, Basel, 1991.

[MT07] N. Mazza and J. Thévenaz. Endotrivial modules in the cyclic case. Arch. Math. (Basel), 89(6):497-503, 2007.

[NR12] G. Navarro and G. R. Robinson. On endo-trivial modules for $p$-solvable groups. Math. Z., 270(3-4):983-987, 2012.

[Rob11] G. R. Robinson. On simple endotrivial modules. Bull. Lond. Math. Soc., 43(4):712-716, 2011.

[RV04] A. Ruiz and A. Viruel. The classification of $p$-local finite groups over the extraspecial group of order $p^{3}$ and exponent p. Math. Z., 248(1):45-65, 2004.

[Suz86] M. Suzuki. Group theory. II, volume 248 of Grundlehren der Mathematischen Wissenschaften [Fundamental Principles of Mathematical Sciences]. Springer-Verlag, New York, 1986. Translated from the Japanese.

[Thé95] J. Thévenaz. G-algebras and modular representation theory. Oxford Mathematical Monographs. The Clarendon Press Oxford University Press, New York, 1995. Oxford Science Publications.

[Wil98] R. A. Wilson. The McKay conjecture is true for the sporadic simple groups. J. Algebra, 207(1):294-305, art. no. ja987450, 1998. 
Caroline Lassueur, FB Mathematik, TU Kaiserslautern, Postfach 3049, 67653 KaisersLAUTERn, GERMANy

E-mail address: lassueur@mathematik.uni-kl.de

Department of Mathematics and Statistics, Lancaster University, Lancaster, LA1 $4 \mathrm{YF}, \mathrm{UK}$

E-mail address: n.mazza@lancaster.ac.uk 